Draft Version November 13, 2018

Preprint typeset using $\mathrm{LT}_{\mathrm{E}} \mathrm{X}$ style emulateapj v. 02/09/03

\title{
DYNAMICAL EVOLUTION OF THIN DISPERSION-DOMINATED PLANETESIMAL DISKS
}

\author{
Roman R. RAFIKOV ${ }^{1,2}$ \& ZaChary S. Slepian ${ }^{1}$ \\ Draft version November 13, 2018
}

\begin{abstract}
We study the dynamics of a vertically thin, dispersion-dominated disk of planetesimals with eccentricities $\tilde{e}$ and inclinations $\tilde{i}$ (normalized in Hill units) satisfying $\tilde{e} \gg 1, \tilde{i} \ll \tilde{e}^{-2} \ll 1$. This situation may be typical for e.g. a population of protoplanetary cores in the end of the oligarchic phase of planet formation. In this regime of orbital parameters planetesimal scattering has an anisotropic character and strongly differs from scattering in thick $(\tilde{i} \sim \tilde{e})$ disks. We derive analytical expressions for the planetesimal scattering coefficients and compare them with numerical calculations. We find significant discrepancies in the inclination scattering coefficients obtained by the two approaches and ascribe this difference to the effects not accounted for in the analytical calculation: multiple scattering events (temporary captures, which may be relevant for the production of distant planetary satellites outside the Hill sphere) and distant interaction of planetesimals prior to their close encounter. Our calculations show that the inclination of a thin, dispersion-dominated planetesimal disk grows exponentially on a very short time scale implying that (1) such disks must be very short-lived and (2) planetesimal accretion in this dynamical phase is insignificant. Our results are also applicable to the dynamics of shear-dominated disks switching to the dispersion-dominated regime.
\end{abstract}

Subject headings:

\section{INTRODUCTION.}

Terrestrial planets are thought to be formed by agglomeration of a large number of primitive rocky or icy bodies known as planetesimals (Safronov 1972). While the origin of planetesimals themselves is still a rather uncertain issue (Youdin 2008) the process of their collisional agglomeration has been extensively explored (Wetherill \& Stewart 1989, 1993; Kenyon \& Luu 1998; Kenyon \& Bromley 2004, 2009). Gravitationally induced bending of the trajectories of interacting bodies called gravitational focusing (Safronov 1972) is known to play a very important role in speeding up the agglomeration process. The degree to which the planetesimal collision rate is amplified by focusing depends sensitively on the velocity dispersion of the planetesimals: the lower is the relative velocity between the interacting bodies the higher are the gravitational focusing and the collision cross-section. Thus, understanding the accretion history of planetesimals is impossible without understanding their dynamical evolution.

Evolution of planetesimal velocities is driven mainly by their mutual gravitational interaction. A convenient way to characterize the shape of planetesimal orbits and their interaction is via the so-called eccentricity and inclination vectors e and i defined as (Ida 1990)

$$
\begin{aligned}
& \mathbf{e}=\left(e_{x}, e_{y}\right)=(e \cos \tau, e \sin \tau), \\
& \mathbf{i}=\left(i_{x}, i_{y}\right)=(i \cos \omega, i \sin \omega),
\end{aligned}
$$

where $e, i, \tau$ and $\omega$ are, respectively, the eccentricity, inclination, and horizontal and vertical phases of the planetesimal. Scattering of two low-mass planetesimals depends only on their relative eccentricity and inclination vectors $\mathbf{e}_{r}=\mathbf{e}_{1}-\mathbf{e}_{2}$ and $\mathbf{i}_{r}=\mathbf{i}_{1}-\mathbf{i}_{2}$ (the so-called Hill approximation, see Hénon \& Petit 1986).

\footnotetext{
1 Department of Astrophysical Sciences, Princeton University, Ivy Lane, Princeton, NJ 08540; rrr@astro.princeton.edu

2 Sloan Fellow
}

There are two important asymptotic regimes of planetesimal interaction: shear-dominated and dispersiondominated. The former is realized when the random component of planetesimal velocity, determined by its eccentricity and inclination, is small compared to the Hill velocity $v_{H}=\Omega R_{H}$. Here $\Omega$ is the local angular frequency in the disk, $R_{H} \equiv a\left(\mu_{1}+\mu_{2}\right)^{1 / 3}$ is the Hill radius, determined by the distance $a$ to the central object and the masses of the interacting bodies $m_{1}$ and $m_{2}$ relative to the central mass $M_{\star}: \mu_{i} \equiv m_{i} / M_{\star}, i=1,2$. Introducing scaled relative eccentricity ${ }^{3} \tilde{\mathbf{e}}_{r}$ and inclination $\tilde{\mathbf{i}}_{r}$ vectors of the interacting bodies as ${ }^{4} \tilde{\mathbf{e}}_{r} \equiv \mathbf{e}_{r} /\left(\mu_{1}+\mu_{2}\right)^{1 / 3}$ and $\tilde{\mathbf{i}}_{r} \equiv \mathbf{i}_{r} /\left(\mu_{1}+\mu_{2}\right)^{1 / 3}$, one can rewrite the condition for the shear-dominated regime as

$$
\tilde{e}_{r}^{2}+\tilde{i}_{r}^{2} \lesssim 1
$$

The relative speed of a pair of interacting bodies in this regime is set mainly by the Keplerian shear.

Dispersion-dominated regime of planetesimal interaction is realized when

$$
\tilde{e}_{r}^{2}+\tilde{i}_{r}^{2} \gtrsim 1
$$

In this case the relative velocity of the planetesimals is determined mainly by their random epicyclic motion while Keplerian shear plays only a minor role. This makes possible analytical treatment of planetesimal dynamics (Ida 1990; Ida \& Makino 1992; Tanaka \& Ida 1996,1997; Stewart \& Ida 2000), which until now has been concentrated on the case when $\tilde{i} \sim \tilde{e}$, so that the random velocity distribution of planetesimals is roughly isotropic. This assumption is very natural in

${ }^{3}$ In this paper all quantities with a tilde are assumed to be scaled by the Hill factor $\left(\mu_{1}+\mu_{2}\right)^{1 / 3}$.

${ }^{4}$ In this paper we use the Hill factor adopted by Hénon \& Petit (1986), which differs by $3^{1 / 3}$ from the scaling used by some other authors. 
advanced stages of dynamical evolution of the dispersiondominated planetesimal population but it may fail in more general situations.

It is thought (Kokubo \& Ida 1998; Rafikov 2004; Goldreich et al. 2004) that at the very end of the oligarchic stage of planetary growth in the inner parts of the Solar System, just before the transition to a chaotic final stage of planetary assembly (sometimes called the stage of giant impacts), planetesimal coagulation produced a number (several hundred) of protoplanetary cores with masses comparable to the mass of the Moon or Mars, i.e. $\sim(0.01-0.1) \mathrm{M}_{\oplus}$. These cores comprised a significant fraction of all the refractory mass of the disk and were well-separated in semi-major axis (typically by several Hill radii).

The orbits of these cores are initially not expected to cross because their eccentricities are very small as a result of efficient dynamical friction exerted on them by the residual population of small planetesimals. However, with time the population of small planetesimals gets eroded by collisional grinding and accretion by cores and the strength of dynamical friction goes down. Distant mutual gravitational perturbations between nearby cores then gradually increase their velocity dispersion, eventually allowing their orbits to cross, which leads to collisions between embryos and their growth into bigger bodies.

Since initially the inclinations of the cores were almost zero distant perturbations cannot efficiently excite vertical motion of cores. Moreover, even though dynamical friction from the remaining planetesimals is no longer efficient in curbing the eccentricity growth of the cores it may still be strong enough to continue damping their inclinations. As a result, the protoplanetary cores are expected to reside in a very thin disk with $\tilde{i} \ll 1$ all the way until the point when their orbits start to cross. When this happens one finds that $\tilde{i} \ll 1 \lesssim \tilde{e}$, so that the condition $\tilde{i} \sim \tilde{e}$ usually assumed in studies of planetesimal dynamical evolution is strongly violated.

Thus, a vertically thin, dispersion-dominated planetesimal disk can naturally arise in some circumstances. Since the collision rate of planetesimals is a sensitive function of their inclination - the smaller is inclination the higher is the collision probability and the faster is protoplanetary growth - it is important to know how much time the population of cores spends in the thin disk configuration after their orbits become crossing. If this time is sufficiently long then core masses could grow significantly by collisions even during the transient period when their inclinations have not yet increased. This possibility potentially may act to speed up the final assembly of terrestrial planets.

A similar situation arises when one considers the transition of the shear-dominated planetesimal population into the dispersion-dominated regime. Ida \& Makino (1992) showed that a planetesimal population starting in the shear-dominated regime typically undergoes a phase in its dynamical evolution when $\tilde{i} \ll 1 \lesssim \tilde{e}$ (see their Fig. 6 for illustration). This phase does not persist for very long but while it lasts the dynamics of the planetesimals are significantly different from the usually assumed case of $\tilde{i} \sim \tilde{e}$.

These considerations give us a motivation to explore the dynamical regime $\tilde{i} \ll 1 \lesssim e$ in this work. The paper is organized as follows: in 92 we describe the equations governing the velocity evolution of the planetesimals while in 3 we analytically compute the scattering coefficients entering these equations in the case of $\tilde{i} \ll 1 \lesssim \tilde{e}$, and in $\$ 4$ we compare our results with numerical calculations. In $\$ 5$ we use our results to examine the velocity evolution of a population of protoplanetary cores with crossing orbits. In 6 we provide comparison with other studies and discuss additional applications of our results.

\section{VELOCITY EVOLUTION.}

To understand the velocity evolution of planetesimals we consider two populations of planetesimals with masses $m_{1}$ and $m_{2}$; populations of different mass contribute linearly to velocity evolution so it is sufficient to consider just two masses. We assume that for every planetesimal type $e_{k}, i_{k} \ll 1$ and $m_{k} \ll M_{\star}, k=1,2$, providing justification for using the Hill approximation.

In this local approximation the Keplerian orbit of a $k$ th planetesimal type is described by the following equations:

$$
\begin{aligned}
x_{k} & =h_{k}-e_{k} \cos \left(t-\tau_{k}\right), \\
y_{k} & =\lambda_{k}-\frac{3}{2} h_{k} t+2 e \sin \left(t-\tau_{k}\right), \\
z_{k} & =i_{k} \sin \left(t-\omega_{k}\right),
\end{aligned}
$$

where $x, y$, and $z$ are Cartesian coordinates in the local radial, azimuthal, and vertical directions centered at some reference stellocentric distance, $h$ is the planetesimal semi-major axis separation from the origin of this coordinate system, and $\lambda$ is a constant related to the origin of time $t$ (measured in units of $\Omega^{-1}$ ).

The relative motion of two non-interacting planetesimals in Hill units $\left(\tilde{\mathbf{r}}_{r}=\left(\mathbf{r}_{1}-\mathbf{r}_{2}\right) / a\left(\mu_{1}+\mu_{2}\right)^{1 / 3}\right)$ is given by equations

$$
\begin{aligned}
& \tilde{x}_{r}=\tilde{h}_{r}-\tilde{e}_{r} \cos \left(t-\tau_{r}\right), \\
& \tilde{y}_{r}=\tilde{\lambda}_{r}-\frac{3}{2} \tilde{h}_{r} t+2 \tilde{e}_{r} \sin \left(t-\tau_{r}\right), \\
& \tilde{z}_{r}=\tilde{i}_{r} \sin \left(t-\omega_{r}\right),
\end{aligned}
$$

where $\tilde{e}_{r}, \tilde{i}_{r}$ are the relative eccentricity and inclination of the planetesimals, $\tilde{h} \equiv\left(a_{1}-a_{2}\right) / a\left(\mu_{1}+\mu_{2}\right)^{1 / 3}$ is the semimajor axes separation normalized in Hill units, and $\tilde{\lambda}_{r}=\left(\lambda_{1}-\lambda_{2}\right) / a\left(\mu_{1}+\mu_{2}\right)^{1 / 3}$. In the following we will drop the subscript " $r$ " from all variables characterizing relative motion of planetesimals where it will not lead to confusion.

Because of the mutual gravitational attraction relative orbital elements appearing in equations (77)-(9) do not remain constant but change according to the following set of equations (Hasegawa \& Nakazawa 1990; Tanaka \& Ida 1996):

$$
\begin{aligned}
& \frac{d \tilde{h}}{d t}=-2 \frac{\partial \phi}{\partial \tilde{y}} \\
& \frac{d \tilde{\lambda}}{d t}=2 \frac{\partial \phi}{\partial \tilde{x}}-3 t \frac{\partial \phi}{\partial \tilde{y}} \\
& \frac{d \tilde{e}_{x}}{d t}=-\sin t \frac{\partial \phi}{\partial \tilde{x}}-2 \cos t \frac{\partial \phi}{\partial \tilde{y}}
\end{aligned}
$$




$$
\begin{aligned}
& \frac{d \tilde{e}_{y}}{d t}=\cos t \frac{\partial \phi}{\partial \tilde{x}}-2 \sin t \frac{\partial \phi}{\partial \tilde{y}}, \\
& \frac{d \tilde{i}_{x}}{d t}=-\cos t \frac{\partial \phi}{\partial \tilde{z}} \\
& \frac{d \tilde{i}_{y}}{d t}=-\sin t \frac{\partial \phi}{\partial \tilde{z}}
\end{aligned}
$$

where

$$
\phi=-\left(\tilde{x}^{2}+\tilde{y}^{2}+\tilde{z}^{2}\right)^{-1 / 2} .
$$

is the interaction potential.

In this work we assume for simplicity that $\mathbf{e}$ and $\mathbf{i}$ of a $k$-th planetesimal population have Gaussian distribution:

$$
\psi\left(\mathbf{e}_{k}, \mathbf{i}_{k}\right) d \mathbf{e}_{k} d \mathbf{i}_{k}=\frac{d \mathbf{e}_{k} d \mathbf{i}_{k}}{4 \pi^{2} \sigma_{e, k}^{2} \sigma_{i, k}^{2}} \exp \left[-\frac{\mathbf{e}_{k}^{2}}{2 \sigma_{e, k}^{2}}-\frac{\mathbf{i}_{k}^{2}}{2 \sigma_{i, k}^{2}}\right],
$$

where $\sigma_{e, k}$ and $\sigma_{i, k}$ are the dispersions of eccentricity and inclination of the $k$-th population. Ida \& Makino (1992) have found that a Gaussian distribution accurately describes the distribution of $\mathbf{e}$ and $\mathbf{i}$ found in direct N-body three-dimensional (3D) simulations of a large number of planetesimals gravitationally interacting in the dispersion-dominated regime. At the same time in their dispersion-dominated simulations of $2 \mathrm{D}$ disks Ida \& Makino found more high-energy particles than a Gaussian distribution would predict. Despite this we still use distribution (17) to represent velocities of planetesimals in thin disks as this is not going to strongly affect the velocity evolution but allows significant simplification.

Our goal is to find how $\sigma_{e, 1}$ and $\sigma_{i, 1}$ of a population with mass $m_{1}$ varies in time as a result of gravitational interactions with planetesimals of mass $m_{2}$ which have eccentricity and inclination dispersions $\sigma_{e, 2}$ and $\sigma_{i, 2}$ (for now we neglect other factors that may affect planetesimal velocities such as gas drag, inelastic collisions, and so on). General evolution equations for the case of distribution (17) have been previously derived by a number of authors (Hornung et al. 1985; Ida 1990; Wetherill \& Stewart 1993; Stewart \& Ida 2000). Here we adopt a specific expression from Rafikov (2003):

$$
\begin{aligned}
& \left.\frac{\partial \sigma_{e, 1}^{2}}{\partial t}\right|_{2}=\frac{3}{4} \Omega N_{2} a^{2}\left(\mu_{1}+\mu_{2}\right)^{4 / 3} \\
& \times\left[\left(\frac{\mu_{2}}{\mu_{1}+\mu_{2}}\right)^{2} H_{1}+2 \frac{\mu_{2}}{\mu_{1}+\mu_{2}} \frac{\sigma_{e, 1}^{2}}{\sigma_{e, 1}^{2}+\sigma_{e, 2}^{2}} H_{2}\right],
\end{aligned}
$$

where $N_{2}$ is the surface number density of bodies with mass $m_{2}$.

Dimensionless stirring coefficients $H_{1,2}$ appearing in equation (18) are defined as

$$
\begin{aligned}
H_{k} & =\int^{\infty} d \tilde{e} d \tilde{i} \tilde{\psi}_{r}(\tilde{e}, \tilde{i}) \hat{H}_{k}(\tilde{e}, \tilde{i}), \quad k=1,2, \\
\hat{H}_{1} & \equiv \int_{-\infty}^{\infty} d \tilde{h}|\tilde{h}|\left\langle(\Delta \tilde{\mathbf{e}})^{2}\right\rangle_{\tau, \omega} \\
\hat{H}_{2} & \equiv \int_{-\infty}^{\infty} d \tilde{h}|\tilde{h}|\langle(\tilde{\mathbf{e}} \cdot \Delta \tilde{\mathbf{e}})\rangle_{\tau, \omega} .
\end{aligned}
$$

Here $\Delta \tilde{\mathbf{e}}$ is the change of $\tilde{\mathbf{e}}$ in the course of scattering, and $\langle\ldots\rangle_{\tau, \omega} \equiv\left(4 \pi^{2}\right)^{-1} \iint d \tau d \omega$ implies averaging over the relative orbital phases characterizing vectors $\tilde{\mathbf{e}}$ and $\tilde{\mathbf{i}}$. Function $\tilde{\psi}_{r}\left(\tilde{e}_{r}, \tilde{i}_{r}\right)$ is the distribution function of relative $\tilde{e}, \tilde{i}$ and can be shown (Stewart \& Ida 2000; Rafikov 2003) to be given by

$$
\psi_{r}(\tilde{e}, \tilde{i}) d \tilde{e} d \tilde{i}=\frac{\tilde{e} d \tilde{e} \tilde{i} d \tilde{i}}{\tilde{\sigma}_{e}^{2} \tilde{\sigma}_{i}^{2}} \exp \left[-\frac{\tilde{e}^{2}}{2 \tilde{\sigma}_{e}^{2}}-\frac{\tilde{i}^{2}}{2 \tilde{\sigma}_{i}^{2}}\right],
$$

where $\tilde{\sigma}_{e}^{2}=\left(\sigma_{e, 1}^{2}+\sigma_{e, 2}^{2}\right) /\left(\mu_{1}+\mu_{2}\right)^{2 / 3}$ and $\tilde{\sigma}_{i}^{2}=\left(\sigma_{i, 1}^{2}+\right.$ $\left.\sigma_{i, 2}^{2}\right) /\left(\mu_{1}+\mu_{2}\right)^{2 / 3}$ are the dispersions of relative eccentricity and inclination. Thus, functions $\hat{H}_{1}$ and $\hat{H}_{2}$ represent scattering coefficients for a planetesimal population with a single value of relative eccentricity $e$ and inclination $i$, while $H_{1}$ and $H_{2}$ are these coefficients averaged over the distribution (22) of $e$ and $i$.

The term inside the brackets in equation (18) proportional to $H_{1}$ is called gravitational stirring (Rafikov 2003) while the term proportional to $\mathrm{H}_{2}$ is called gravitational friction and is different from the "dynamical friction" used by other authors (Stewart \& Ida 2000; Ohtsuki et al. 2002).

Equation (18) also describes the self-stirring of population with mass $m_{1}$ if one changes the subscript " 2 " to " 1 " in its right hand side (which makes expression in brackets equal to $\left.\left(H_{1}+2 H_{2}\right) / 4\right)$. For a continuous distribution of planetesimal masses equation (18) should be generalized by integrating the right hand side over the mass spectrum.

Equations analogous to (18) can be written for the inclination evolution by replacing "e" by "i" everywhere in equations (18)-(21) and using scattering coefficients $\hat{K}_{1,2}$ and $K_{1,2}$ defined analogously to expressions (19)-(21) instead of $\hat{H}_{1,2}$ and $H_{1,2}$ ).

\section{SCATTERING COEFFICIENTS.}

System (18)-(21) is a rather general set of equations derived for a Gaussian distribution of orbital elements (17). It allows one to determine how $\sigma_{e, k}$ and $\sigma_{i, k}(k=$ $1,2)$ evolve in time once coefficients $\tilde{H}_{1,2}$ and $\tilde{K}_{1,2}$ are known as functions of $\tilde{\sigma}_{e}$ and $\tilde{\sigma}_{i}$. These coefficients have been previously calculated by a number of authors in the dispersion-dominated case under the assumption that $\tilde{i} \sim$ $\tilde{e}$. However, as we demonstrate shortly, these calculations become invalid once $\tilde{i}$ gets sufficiently small while $\tilde{e} \gg 1$. Thus our next step is to rederive scattering coefficients in the case of $\tilde{i} \ll \tilde{e}$ from the first principles.

In doing so we will adopt an approach previously developed by Ida et al. (1993) for the dispersion-dominated regime. According to this method (1) the approach trajectory of one planetesimal far from another is represented as a straight line and (2) the effect of perturbations from the central mass on the gravitational scattering of the two planetesimals is neglected. There is also an implicit assumption that (3) the scattering coefficients are dominated (or at least strongly contributed to) by those planetesimals whose trajectories pass very close to the perturber. If the latter assumption is fulfilled then the other two are quite natural. Indeed, the first approximation works well because most of the perturbation to the orbit of the passing particle occurs near the point of closest approach of the interacting bodies. In this region the curvature of the planetesimal trajectory 


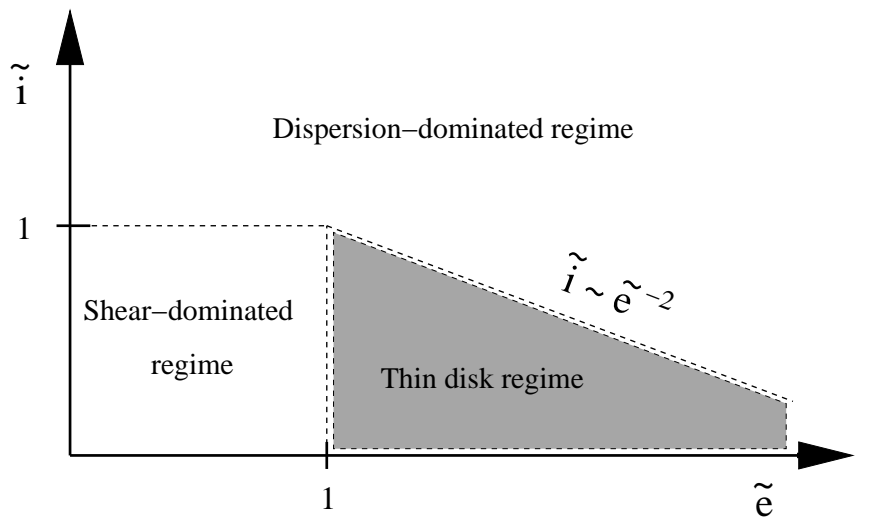

FIG. 1.- Schematic illustration of different regions in the $\tilde{e}$ $\tilde{i}$ phase space, showing the shear- and dispersion-dominated regions and the thin-disk dynamical regime (shaded), which is also dispersion-dominated $(\tilde{e} \gtrsim 1)$.

caused by epicyclic motion can be neglected, justifying the straight-line simplification. The second approximation works because the most significant contribution to scattering is due to trajectories passing very close to the perturber, within its Hill radius, where the influence of the third, central body can be disregarded.

When $\tilde{e} \sim \tilde{i} \gg 1$ the flux of approaching planetesimals in the vicinity of any given perturber is roughly uniform on scales $\sim R_{H}$, and every decade in the initial impact parameter of interacting bodies contributes roughly equally to the scattering coefficients (Binney \& Tremaine 1987). This gives rise to appearance of the so-called Coulomb logarithm, $\ln \Lambda$, in expressions for the scattering coefficients. The argument $\Lambda$ is roughly the ratio of the maximum impact parameter $l_{\max } \sim \tilde{i} R_{H}$, beyond which the density of approaching planetesimals is non-uniform, to the impact parameter

$$
l_{\text {min }} \sim \frac{R_{H}}{\tilde{e}^{2}+\tilde{i}^{2}},
$$

at which the trajectories of incoming planetesimals experience large-angle deflection.

Previous calculations of the scattering coefficients in the dispersion-dominated regime assumed that $l_{\max } \gg$ $l_{\text {min }}$ meaning that $\ln \Lambda \gtrsim 1$. In this case the scattering calculation for closely approaching orbits with impact parameters $\sim l_{\min } \ll R_{H}$ which can be done analytically approximates quite well (with logarithmic accuracy) the full scattering coefficients so that the aforementioned assumption of the dominance of close encounters for scattering coefficient calculation is roughly fulfilled. Clearly, $l_{\max } \gg l_{\min }$ requires that $\tilde{i} \gg 1 / \tilde{e}^{2}$, which is essentially a condition for the standard expressions for the dispersiondominated scattering coefficients to be valid.

In this work we look at the opposite extreme, namely a situation when

$$
\tilde{i} \lesssim \tilde{i}_{c r i t} \equiv \tilde{e}^{-2} \ll 1, \quad \tilde{e} \gg 1,
$$

(see Figure 1 for illustration). When this condition is satisfied the assumption of a uniform distribution of approaching planetesimals around the scatterer does not hold even for $l \sim l_{\text {min }}$. Then a new calculation of scattering coefficients is needed.

In Appendix A we present the details of such a calculation which makes the following set of assumptions: (1) the planetesimals move at high relative velocities which allows us to use a two-body scattering approximation, (2) the planetesimal velocities change only during close approaches, which are possible only when $\tilde{h}<\tilde{e}$, (3) the gravitational interaction between planetesimals at large separations is neglected, and (4) after changing as a result of the encounter with the scatterer the planetesimal's orbital elements do not change further. These simplifications allow us to derive the following set of expressions for the integrands of the scattering coefficients, see equations (20)-(21):

$$
\begin{aligned}
\left\langle(\Delta \tilde{\mathbf{e}})^{2}\right\rangle_{\omega, \tau} & =\frac{20}{3} \frac{\tilde{v}}{|\tilde{h}| \sqrt{\tilde{e}^{2}-\tilde{h}^{2}}}, \\
\langle\tilde{\mathbf{e}} \cdot \Delta \tilde{\mathbf{e}}\rangle_{\omega, \tau} & =-\frac{4}{3} \frac{\tilde{e}^{2}}{\tilde{v}|\tilde{h}| \sqrt{\tilde{e}^{2}-\tilde{h}^{2}}}, \\
\left\langle(\Delta \tilde{\mathbf{i}})^{2}\right\rangle_{\omega, \tau} & =\frac{2}{3} \frac{\tilde{v}^{5} \tilde{i}^{2}}{|\tilde{h}| \sqrt{\tilde{e}^{2}-\tilde{h}^{2}}} . \\
\langle\tilde{\mathbf{i}} \cdot \Delta \tilde{\mathbf{i}}\rangle_{\omega, \tau} & =-\frac{2}{3} \frac{\tilde{i}^{2}}{\tilde{v}|\tilde{h}| \sqrt{\tilde{e}^{2}-\tilde{h}^{2}}},
\end{aligned}
$$

Note that these expressions do not contain a Coulomb logarithm and do not suffer from the ambiguity related to the choice of minimum and maximum values of impact parameter $l_{\min }$ and $l_{\max }$ typical for the $3 \mathrm{D}$ case. The physical reason for this lies in the fact that in the limit (24) the scattering coefficients are dominated by trajectories with impact parameters $l \sim l_{\text {min }}$, i.e. those leading to large-angle scattering. Thus, integrals over $d l$ appearing in the calculation of the scattering coefficients are mostly contributed to by $l \sim l_{\min }$ in the quasi-2D case $^{5}$, so that values of $l$ much larger and much smaller than $l_{\min }$ affect the coefficients only weakly. This is very different from the 3D case, in which trajectories experiencing weak scattering provide significant contribution to the scattering coefficients.

Integrating these equations over $|\tilde{h}| d \tilde{h}$ from 0 to $\tilde{e}$ (limits within which a given planetesimal can experience a close encounter with the scatterer) and substituting into equations (20)-(21) we arrive at the following expressions for the scattering coefficients corresponding to fixed $\tilde{e}$ and $\tilde{i}$ and averaged over the phase angles $\tau$ and $\omega$ :

$$
\begin{aligned}
& \hat{H}_{1}=\frac{40}{3} \mathbf{E}\left(\frac{\sqrt{3}}{2}\right) \tilde{e}_{r}=16.147 \tilde{e}_{r}, \\
& \hat{H}_{2}=-\frac{8}{3} \mathbf{K}\left(\frac{\sqrt{3}}{2}\right) \tilde{e}_{r}=-5.751 \tilde{e}_{r}, \\
& \hat{K}_{1}=\frac{1}{9}\left[\frac{41}{5} \mathbf{E}\left(\frac{\sqrt{3}}{2}\right)-\mathbf{K}\left(\frac{\sqrt{3}}{2}\right)\right] \tilde{i}_{r}^{2} \tilde{e}_{r}^{5} \\
& =0.864 \tilde{i}_{r}^{2} \tilde{e}_{r}^{5}, \\
& \hat{K}_{2}=-\frac{4}{3} \mathbf{K}\left(\frac{\sqrt{3}}{2}\right) \frac{\tilde{i}_{r}^{2}}{\tilde{e}_{r}}=-2.875 \frac{\tilde{i}_{r}^{2}}{\tilde{e}_{r}} .
\end{aligned}
$$

Finally, averaging coefficients (29)-(32) over the Gaus-

\footnotetext{
${ }^{5}$ Note that this property makes the assumptions adopted in our calculation quite robust.
} 
sian distribution (17) one finds that

$$
\begin{aligned}
H_{1} & =\frac{20 \sqrt{2 \pi}}{3} \mathbf{E}\left(\frac{\sqrt{3}}{2}\right) \tilde{\sigma}_{e, r} \approx 20.237 \tilde{\sigma}_{e, r}, \\
H_{2} & =-\frac{4 \sqrt{2 \pi}}{3} \mathbf{K}\left(\frac{\sqrt{3}}{2}\right) \tilde{\sigma}_{e, r} \approx-7.207 \tilde{\sigma}_{e, r} \\
K_{1} & =\frac{5 \sqrt{2 \pi}}{3}\left[\frac{41}{5} \mathbf{E}\left(\frac{\sqrt{3}}{2}\right)-\mathbf{K}\left(\frac{\sqrt{3}}{2}\right)\right] \tilde{\sigma}_{i, r}^{2} \tilde{\sigma}_{e, r}^{5} \\
& \approx 32.478 \tilde{\sigma}_{i, r}^{2} \tilde{\sigma}_{e, r}^{5}, \\
K_{2} & =-\frac{4 \sqrt{2 \pi}}{3} \mathbf{K}\left(\frac{\sqrt{3}}{2}\right) \frac{\tilde{\sigma}_{i, r}^{2}}{\tilde{\sigma}_{e, r}} \approx-7.207 \frac{\tilde{\sigma}_{i, r}^{2}}{\tilde{\sigma}_{e, r}}
\end{aligned}
$$

These expressions represent the behavior of scattering coefficients in the limit (24).

\section{COMPARISON WITH NUMERICAL RESULTS.}

To check our analytical results we ran a series of numerical calculations. The latter are the Monte-Carlo computations of integrals in equations (19)-(21) with $\Delta \tilde{\mathbf{e}}_{r}$, $\Delta \tilde{\mathbf{i}}_{r}$ obtained by direct integration of equations (10)-(15). Equations for the evolution of orbital elements have been integrated using fourth-order Runge-Kutta method with adaptive step size control (Press et al. 1992). Conservation of Jacobi constant has been routinely monitored and this integral of motion has been found to vary during the calculation by at most one part in $10^{5}$ for a very small number of orbits. In the majority of calculations the Jacobi constant has been conserved to relative accuracy of better than $10^{-10}$.

The orbits used in the Monte-Carlo evaluation of integrals have been drawn from the distribution of initial orbital parameters appropriate for each particular scattering coefficient. When computing $\langle\tilde{\mathbf{e}} \cdot \Delta \tilde{\mathbf{e}}\rangle_{\omega, \tau},\left\langle(\Delta \tilde{\mathbf{e}})^{2}\right\rangle_{\omega, \tau}$, etc. we select a set of values of $\tilde{e}, \tilde{i}$, and $\tilde{h}$, and draw $\tau$ and $\omega$ randomly from a uniform distribution between 0 and $2 \pi$. When computing $\hat{H}_{1,2}, \hat{K}_{1,2}$ we also draw $\tilde{h}$ from a uniform distribution between $-L_{h}$ and $L_{h}$, while keeping $\tilde{e}, \tilde{i}$ fixed. Finally, to compute $H_{1,2}, K_{1,2}$ we draw $\tilde{e}_{x}, \tilde{e}_{y}, \tilde{i}_{x}, \tilde{i}_{y}$ randomly from a Gaussian distribution (17) with given dispersions $\tilde{\sigma}_{e}$ and $\tilde{\sigma}_{i}$, while $\tilde{h}$ is drawn from a uniform distribution between $-L_{h}$ and $L_{h}$.

In all our of calculations of $\hat{H}_{1,2}, \hat{K}_{1,2}$ we use

$$
L_{h}=10+4 \tilde{e},
$$

to ensure that even orbits with $\tilde{h}>\tilde{e}$ are properly accounted for. When computing $H_{1,2}, K_{1,2}$ we use the same prescription for $L_{h}$ but with $\tilde{\sigma}_{e}$ replacing $\tilde{e}$. We adopted the following prescription for the number of orbits used for evaluating scattering coefficients:

$$
N=5 \times 10^{4}\left[10+(1+\tilde{i})(1+\tilde{e})^{2}\right] .
$$

Thus, to compute scattering coefficients for $\tilde{e}=15$ we run around 13 million scattering calculations. For $H_{1,2}$, $K_{1,2}$ we used the same prescription with $\tilde{\sigma}_{e}, \tilde{\sigma}_{i}$ replacing $\tilde{e}, \tilde{i}$.

In Figure 2 we present the results of calculation of $\hat{H}_{1,2}, \hat{K}_{1,2} / \tilde{i}^{2}$ as a function of $\tilde{e}$, for several values of $\tilde{i}=10^{-1}, 10^{-2}, 10^{-4}, 10^{-6}$, together with our analytical
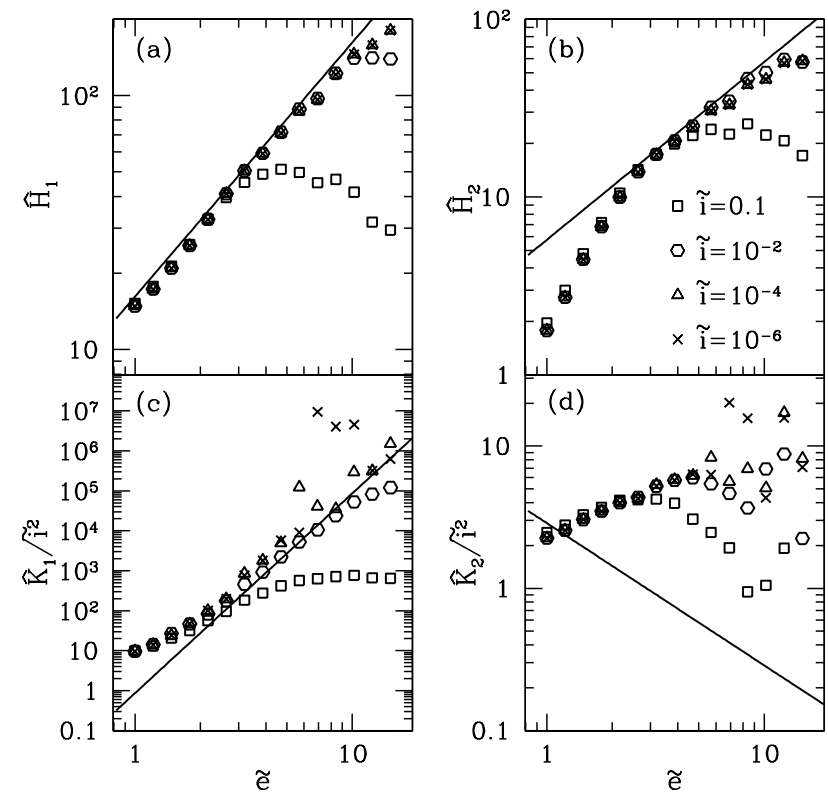

FIG. 2.- Results of numerical calculation of scattering coefficients (a) $\hat{H}_{1}$, (b) $\hat{H}_{2}$, (c) $\hat{K}_{1} / \tilde{i}^{2}$, (d) $\hat{K}_{2} / \tilde{i}^{2}$, compared with analytical predictions (29)-(32), represented by solid lines. Values of coefficients are shown as functions of $\tilde{e}$ for $\tilde{i}=10^{-1}, 10^{-2}, 10^{-4}, 10^{-6}$ (see legend in panel (b) for associating different dot styles with particular $\tilde{i}$ )
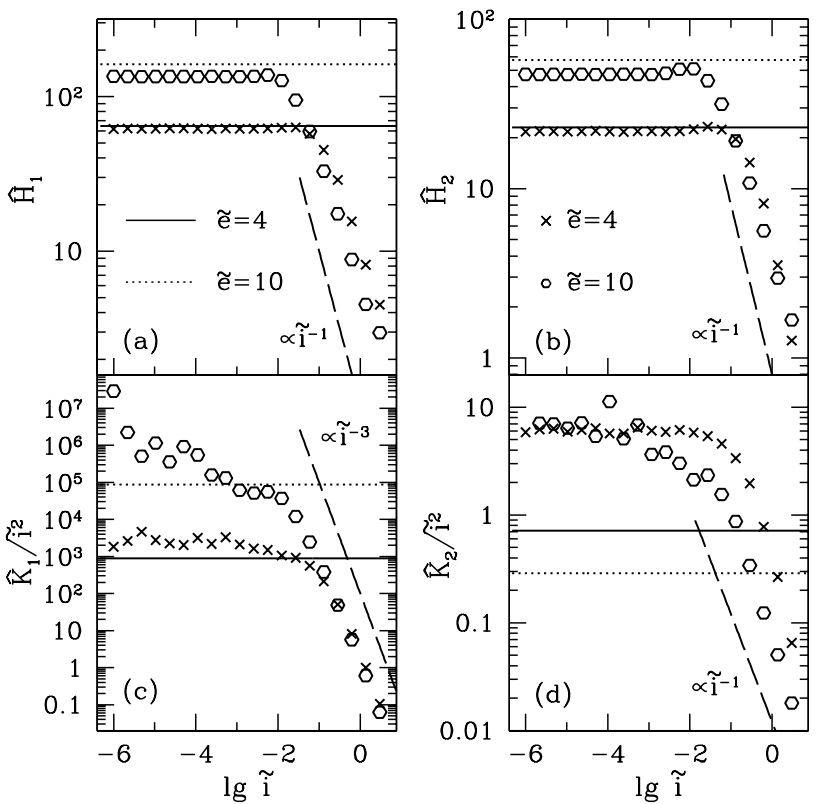

FIG. 3.- Plots of the same scattering coefficients as in Figure 2 but now as a function of $\tilde{i}$ for $\tilde{e}=4$ and $\tilde{e}=10$ (see legend in panel (b)). Analytical results for the case of a thin disk are shown as solid lines for $\tilde{e}=4$ and as dotted lines for $\tilde{e}=10$. Long-dashed lines show analytical scaling of scattering coefficients in 3D regime, when $\tilde{i} \gtrsim \tilde{e}^{-2}$ 
predictions (29)-(32) shown by solid lines. Values of $\hat{K}_{1,2}$ are scaled by $\tilde{i}^{2}$ to simplify comparison of curves corresponding to different $\tilde{i}$.

Figure 21 a demonstrates rather good agreement between analytical and numerical results for the gravitational stirring coefficient $\hat{H}_{1}$ almost everywhere in the considered range. Agreement at very small values of $\tilde{e}$ is likely a coincidence since at $\tilde{e} \approx 1$ a transition to a sheardominated scattering should occur which invalidates our assumption of $\tilde{e} \gg 1$. At higher values of $\tilde{e}$ curves corresponding to different $\tilde{i}$ generally line up with the analytical results quite well except for the noticeable deviation of $\tilde{i}=0.1$ curve from analytical result (29) which starts around $\tilde{e} \approx 3$ and becomes stronger as $\tilde{e}$ grows. This deviation is expected since our analytical results are valid only for $\tilde{i}$ satisfying the constraint (24). For $\tilde{i}=0.1$ this means that agreement with the analytical result is expected only for $\tilde{e} \lesssim \tilde{i}^{-1 / 2} \approx 3$, in good correspondence with Figure 2 This point is reinforced by observing the deviation of $\tilde{i}=10^{-2}$ results from the analytical curve that starts at $\tilde{e} \approx 10 \approx\left(10^{-2}\right)^{1 / 2}$, i.e. again agrees with the constraint (24). Points for $\tilde{i}=10^{-4}$ and $10^{-6}$ fall on top of each other in the whole range of calculation as they should. They lie somewhat below the analytical prediction for large values of $\tilde{e}$, which we do not have a good explanation for.

The same applies very well to the results for the gravitational friction coefficient $\hat{H}_{2}$ shown in Figure 2b. The only slight difference is that the influence of the sheardominated regime is more pronounced for this scattering coefficient, as $\hat{H}_{2}$ settles onto the analytical result (30) only at $\tilde{e} \approx 2.5$. Thus, the scattering coefficients based on eccentricity changes agree with theory quite well within the range of applicability of the analytical results.

We now turn to coefficients $\hat{K}_{1,2}$ which are based on changes in inclination. As one can see from Figure $2 k$, the shear-dominated regime affects stirring coefficient $\hat{K}_{1}$ for $\tilde{e} \lesssim 2.5$. As expected from our previous discussion, $\hat{K}_{1}$ strongly deviates from the analytical prediction starting at around $\tilde{e} \approx 3$ for $\tilde{i}=0.1$ and at around $\tilde{e} \approx 10$ for $\tilde{i}=10^{-2}$. However, the results for $\tilde{i}=10^{-4}$ and $\tilde{i}=10^{-6}$ generally do not fall on top of each other as one would expect given that the quadratic scaling of $\hat{K}_{1,2}$ with $\tilde{i}$ has been removed in Figures $2 \mathrm{k}$,d. Moreover, the values of $\hat{K}_{1}$ clearly deviate quite strongly from the analytical prediction (31), sometimes by three orders of magnitude, without any recognizable regular pattern.

The numerical results for the gravitational friction coefficient $K_{2}$ as compared with theory are even more surprising, as Figure $2 \mathrm{~d}$ demonstrates. Here, for small values of $\tilde{e} \lesssim 5, \hat{K}_{2}$ systematically increases with $\tilde{e}$, while analytical result (32) predicts that $\hat{K}_{2}$ should be a decreasing function of $\tilde{e}$. At larger $\tilde{e}$ numerically computed values of $\hat{K}_{2}$ exhibit significant scatter in a chaotic fashion. To be fair, one should note that some of the theoretical expectations are confirmed by numerics even for $\hat{K}_{2}$ : a curve for $\tilde{i}=0.1$ again diverges from other curves corresponding to smaller values of $\tilde{i}$ at $\tilde{e} \approx 3$.

In Figure 3 we look at the behavior of scattering coefficients as functions of $\tilde{i}$ for a fixed value of $\tilde{e}$. The main
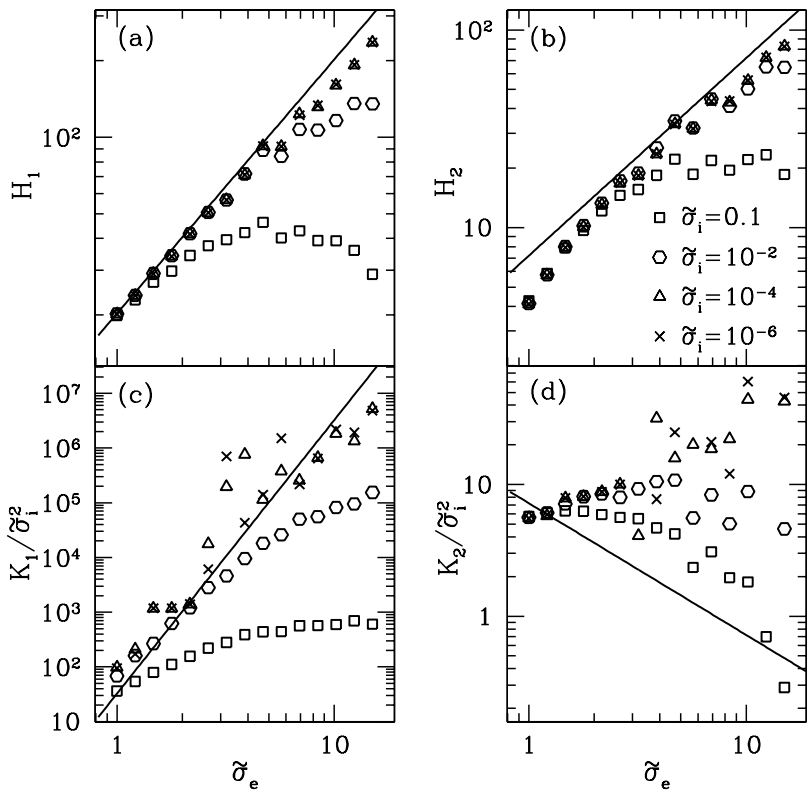

Fig. 4.- Same as Figure 2 but for scattering coefficients (a) $H_{1}$, (b) $H_{2}$, (c) $K_{1} / \tilde{\sigma}_{i}^{2}$, (d) $K_{2} / \tilde{\sigma}_{i}^{2}$, corresponding to the Gaussian distribution of $\tilde{e}$ and $\tilde{i}$. Coefficients are shown as a function of $\tilde{\sigma}_{e}$ for several values of $\tilde{\sigma}_{i}=10^{-1}, 10^{-2}, 10^{-4}, 10^{-6}$ (see legend in panel (b) for associating different dot styles with different $\tilde{\sigma}_{i}$ )
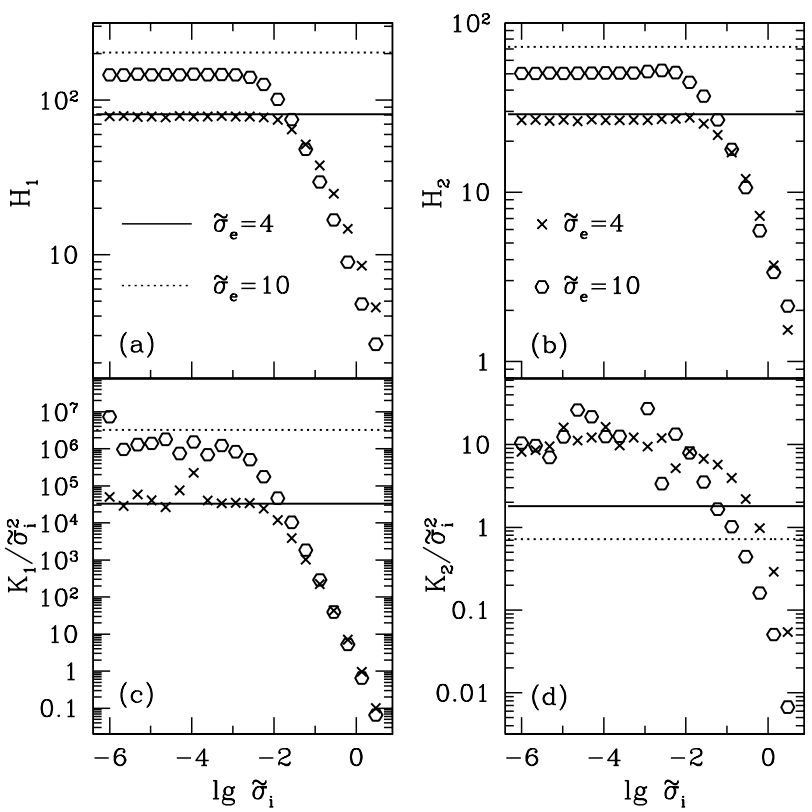

FIG. 5.- Plots of the same scattering coefficients as in Figure 4 as a function of $\tilde{\sigma}_{i}$ for $\tilde{\sigma}_{e}=4$ and $\tilde{\sigma}_{e}=10$ (see legend in panel (b)). Analytical results for the case of thin disk are shown as solid lines for $\tilde{\sigma}_{e}=4$ and as dotted lines for $\tilde{\sigma}_{e}=10$.

goal of these plots is to illustrate the transition between the thin and thick disk regimes of planetesimal scattering occuring at $i_{\text {crit }} \approx \tilde{e}^{-2}$. The rather good accuracy of our analytical results can be clearly seen in the behavior of $\hat{H}_{1,2}$ and even $\hat{K}_{1}$ (the situation is less clear in the case of $\hat{K}_{2}$ ): the behavior of the scattering coefficients changes 
dramatically at $\tilde{i} \approx 10^{-2}$ for $\tilde{e}=10$ and at $\tilde{i} \approx 0.05$ for $\tilde{e}=4$. The long-dashed lines in Figure 3 illustrate the scaling of the scattering coefficients with $\tilde{i}$ in the thickdisk regime (Stewart \& Ida 2000), and show good agreement with our numerical results when the condition (24) is violated. It is clear from Figures $3 \mathrm{~b}, \mathrm{~b}$ that our thindisk theory describes the behavior of eccentricity-based coefficients $\hat{H}_{1,2}$ quite accurately even for $\tilde{e}=4$, which is not very far from the shear-dominated regime.

However, from Figures 3 r,d one sees once again that the inclination-based coefficients $\hat{K}_{1,2}$ deviate from analytical predictions. Already for $\tilde{e}=4$ coefficient $\hat{K}_{1}$ exhibits stochastic variations as a function of $\tilde{i}$ by a factor of order unity. At $\tilde{e}=10$ these variations become quite dramatic and exhibit an increasing trend with decreasing $\tilde{i}$. This is rather surprising since one expects analytical theory to work better for very small values of $\tilde{i}$, when the condition (24) is satisfied by a large margin. This clearly indicates that the theory is missing some important ingredient, a conclusion which is additionally reinforced by Figure $3 \mathrm{~d}$ demonstrating rather poor agreement between numerical and analytical values of $\hat{K}_{2}$.

In Figures 4, 5 we show the behavior of scattering coefficients $H_{1,2}, K_{1,2}$ averaged over the Gaussian distribution of $\tilde{e}$ and $\tilde{i}$. As expected, all the major features of $\hat{H}_{1,2}, \hat{K}_{1,2}$ discussed above are preserved in these plots, although the overall agreement with theory is additionally spoiled by the fact that numerically computed $H_{1,2}$, $K_{1,2}$ represent a Gaussian convolution of $\hat{H}_{1,2}, \hat{K}_{1,2}$ over an extended range in $\tilde{e}$ and $\tilde{i}$, and not everywhere inside this range are the basic assumptions (e.g. dispersiondominated scattering) of our analytical theory fulfilled. In particular, numerical coefficients are affected to some extent by shear-dominated scattering events, not accounted for in our theory. Also, at high $\tilde{\sigma}_{i} \sim 0.1-10^{-2}$ a significant fraction of numerically integrated scattering events had values of $\tilde{i} \sim \tilde{e}$ corresponding to thick disk scattering, for which the behavior of coefficients is different from our theory; see Figure 3 .

Based on the results presented in Figures 2 25 we conclude that analytical theory explains quite well the behavior of scattering coefficients based on changes of $\tilde{e}$, while it provides a rather poor fit to the numerically determined behavior of the inclination-based scattering coefficients. There may be several reasons for this discrepancy, some of which are listed below.

1. It may be that the discrepancy arises when we integrate the phase-averaged coefficients $\langle\tilde{\mathbf{e}} \cdot \Delta \tilde{\mathbf{e}}\rangle_{\omega, \tau}$, $\left\langle(\Delta \tilde{\mathbf{e}})^{2}\right\rangle_{\omega, \tau}$, etc. over $\tilde{h}$ to obtain $\hat{H}_{1,2}$, etc.; see definitions (20) and (21). In particular, encounters with $\tilde{h}>\tilde{e}$ neglected in our analytical work may provide an important contribution to the numerically computed rates.

2. The two-body approximation used in our analytical calculations does not work well.

3. Our assumption of a single close scattering per approaching orbit may be faulty, as the scattered planetesimals may have orbital parameters allowing them to experience additional close approaches with the scatterer.

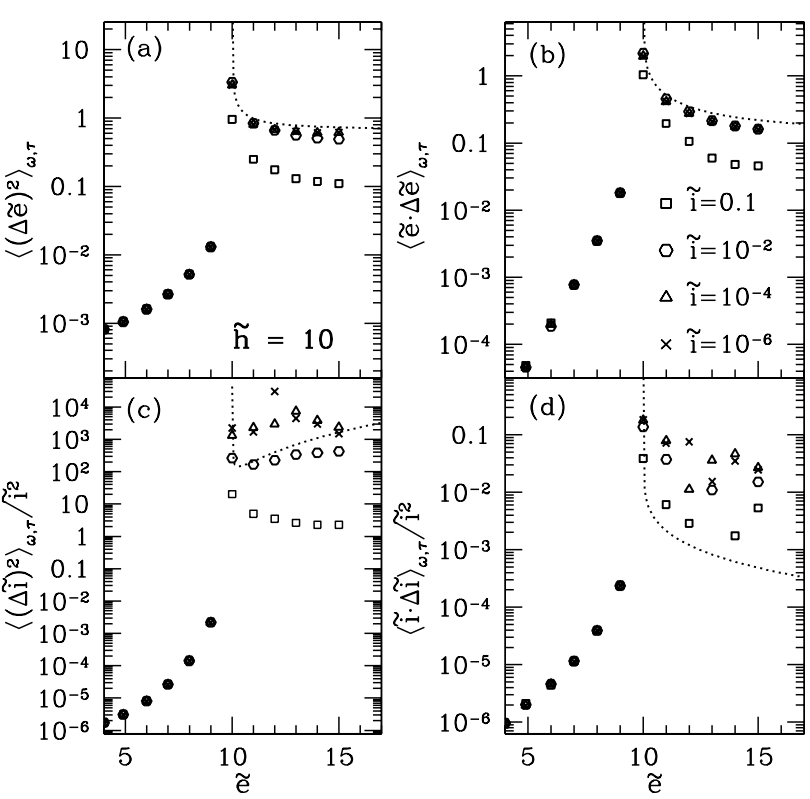

FIG. 6. - Plots of phase-averaged scattering coefficients (a) $\left\langle(\Delta \tilde{\mathbf{e}})^{2}\right\rangle_{\omega, \tau}, \quad$ (b) $\langle\tilde{\mathbf{e}} \cdot \Delta \tilde{\mathbf{e}}\rangle_{\omega, \tau}$, (c) $\left\langle(\Delta \tilde{\mathbf{i}})^{2}\right\rangle_{\omega, \tau}$, (d) $\langle\tilde{\mathbf{i}} \cdot \Delta \tilde{\mathbf{i}}\rangle_{\omega, \tau}$, as functions of $\tilde{e}$ for a fixed value of $\tilde{h}=10$ and several values of $\tilde{i}=0.1,10^{-2}, 10^{-4}, 10^{-6}$; see legend in panel (b). Note the rapid decay of scattering coefficients for $\tilde{e}<\tilde{h}$. Dotted lines show analytical predictions for $\tilde{e}>\tilde{h}$.

4. Our theory assumes that the changes in planetesimal orbital elements occur only during the close approach, when the planetesimal separation is $\lesssim R_{H}$, while in reality it may be that the more distant interactions between planetesimals at separations $\gtrsim R_{H}$ also play an important role.

We devote the rest of this section to exploring these possibilities.

\subsection{Integration over $\tilde{h}$.}

To figure out whether the aforementioned discrepancy between the analytical and numerical inclination-based scattering coefficients can be caused by the integration of the phase-averaged coefficients over $\tilde{h}$ we look at the behavior of the phase-averaged coefficients. In Figure [6] we present their scaling with $\tilde{e}$ for a fixed value of $\tilde{h}=10$ and several values of $\tilde{i}$. Similarly, in Figure 7 these coefficients are shown as functions of $\tilde{h}$ for fixed $\tilde{e}$ and the same values of $\tilde{i}$. Based on these plots we can make several conclusions.

First, when $\tilde{e}>\tilde{h}$ analytical predictions for $\langle\tilde{\mathbf{e}} \cdot \Delta \tilde{\mathbf{e}}\rangle_{\omega, \tau}$ and $\left\langle(\Delta \tilde{\mathbf{e}})^{2}\right\rangle_{\omega, \tau}$ fit our numerical results quite well. Coefficients computed for $\tilde{i}=0.1$ deviate from theory because, as previously described, they do not correspond to the thin disk scattering regime. At the same time $\langle\tilde{\mathbf{i}} \cdot \Delta \tilde{\mathbf{i}}\rangle_{\omega, \tau}$ and $\left\langle(\Delta \tilde{\mathbf{i}})^{2}\right\rangle_{\omega, \tau}$ are still significantly different from theory and exhibit rather erratic behavior.

Second, the values of all scattering coefficients corresponding to $\tilde{e}<\tilde{h}$ are much smaller than their values for $\tilde{e}>\tilde{h}$. In the latter case planetesimals can experience a close approach, while in the former this is not possible, 

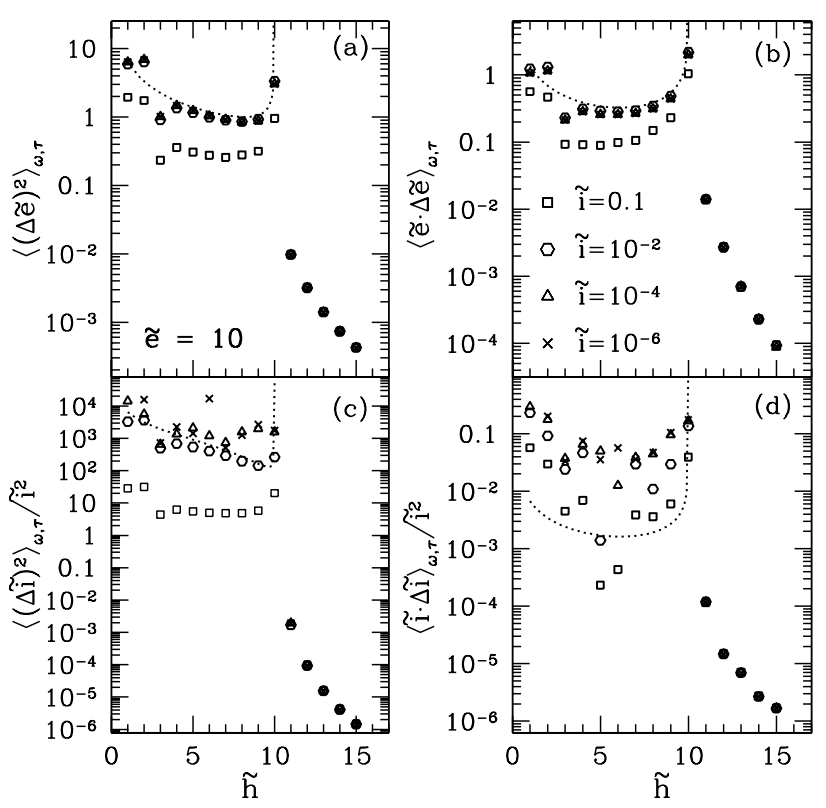

FIG. 7.- Same as Figure 7 but with phase averaged coefficients plotted as functions of $\tilde{h}$ for $\tilde{e}=10$.

and changes of orbital elements are much weaker than in the latter case. As a result, contribution of orbits with $\tilde{e}<\tilde{h}$ to scattering coefficients is very small. In fact, one can see from Figure 7 that for $\tilde{e}=10,\left\langle(\Delta \tilde{\mathbf{e}})^{2}\right\rangle_{\omega, \tau}$ computed at $\tilde{h}=10$ (close encounters possible) and $\tilde{h}=11$ (close encounters not possible) differ by more than 2 orders of magnitude. The same is true for $\langle\tilde{\mathbf{e}} \cdot \Delta \tilde{\mathbf{e}}\rangle_{\omega, \tau}$, while for $\left\langle(\Delta \tilde{\mathbf{i}})^{2}\right\rangle_{\omega, \tau}$ and $\langle\tilde{\mathbf{i}} \cdot \Delta \tilde{\mathbf{i}}\rangle_{\omega, \tau}$ this difference is 6 and 3 orders of magnitude respectively. This very well illustrates our point made in $\$ 3$ that trajectories experiencing large-angle scattering (possible only for $\tilde{e}>\tilde{h}$ ) strongly dominate scattering coefficients in the case of a thin planetesimal disk.

To summarize, the results displayed in Figures 6]:7] make it clear that the well-separated orbits with $\tilde{e}<\tilde{h}$, for which strong scattering is impossible, do not contribute much to the scattering coefficients. All the discrepancy between the theoretical and numerical values of $\hat{K}_{1,2}$ and $K_{1,2}$ is already present in the corresponding phase-averaged coefficients, and is not introduced by the integration of the phase-averaged coefficients over only a finite range of $\tilde{h},|\tilde{h}|<\tilde{e}$.

\subsection{Accuracy of the two-body approximation.}

Next we consider whether the two two-body approximation adopted in our analytical calculations is valid for the case of thin disk scattering.

Previously, Tanaka \& Ida (1996) have compared numerically computed changes of orbital parameters resulting from gravitational scattering with analytical predictions derived in the two-body approximation. They found good agreement between the two, except for the narrow regions of the initial epicyclic phases in which orbital parameters evolved in a chaotic manner, if (a) $\tilde{h} \gtrsim 2$, (b) the encounter velocity $\tilde{v}_{0} \gtrsim 4$ and (c) a small shift in the initial epicyclic phases $\tau$ and $\omega$ is introduced to match analytical predictions. Tanaka \& Ida (1996) have compared only $\Delta h$ calculated by both methods for $\tilde{i}=0$, and also the changes of other orbital elements for $\tilde{i} \sim \tilde{e} \gtrsim 1$. None of these cases corresponds to the regime of thin disk scattering considered in this work although the former does describe quite well the variation of the eccentricity-based scattering coefficients. For that reason we ran our own calculations with initial orbital parameters selected to correspond to the thin disk case.

In general we find good agreement with the conclusions of Tanaka \& Ida (1996), as shown in particular in Figure 8 where we display the changes of various orbital elements resulting from gravitational scattering as well as the minimum approach distance between the scattering bodies $\tilde{l}_{\text {min }}$. In making this Figure we have slightly shifted analytical curves (shown as dotted lines) in $\tau$ by $\Delta \tau=-0.05$ to make them better match numerical results (shown as solid curves). In practice such a shift of the orbital phase arises due to the distant interaction between the planetesimals as they approach each other (Tanaka \& Ida 1996). Only one interval of $\tau$ in which strong scattering is possible is shown, $0.3<\tau<0.39$; another one exists at $5.97<\tau<6.05$, in accordance with the discussion in Appendix, where the existence of two values of $\tau$ for which close encounters are possible for $\tilde{e}>\tilde{h}$ is stated.

One can deduce from Figure 8 that analytical curves follow the numerical results quite well for the majority of values of $\tau$ except for the two narrow ranges of $\tau$, namely $0.32<\tau<0.33$ and $0.35<\tau<0.355$. Inside these intervals orbital elements experience strong chaotic variations as $\tau$ changes, with $\Delta \tilde{h}, \Delta \tilde{e}_{x}, \Delta \tilde{e}_{y}$ deviating from analytical prediction by a factor of order unity, while $\Delta \tilde{i}_{x}, \Delta \tilde{i}_{y}$ differ from theory by several orders of magnitude (off scale on these plots)! Although these deviations are very significant we will show next that they are not caused by the failure of the two-body approximation. Thus, use of the two-body approximation cannot explain the discrepancy between the numerical and analytical inclinationbased scattering coefficients.

\subsection{Single-scattering approximation.}

Our calculation has always assumed that changes of orbital elements arising during a scattering event are final. In reality there may be a situation when a post-scattering orbital elements are such that they cause another close approach between planetesimals, leading to additional variation of orbital elements. And this may happen not just once for a given incoming orbit. Such multiple scattering events are very typical for planetesimals scattering in the shear-dominated regime but their importance in the high-velocity case is not very obvious.

To see that multiple scattering is indeed possible even in the dispersion-dominated regime we take a closer look at Figure 8 where we plot $\Delta \tilde{h}$ as a function of $\tau$. One can see that chaotic orbits strongly deviating from analytical prediction (shown as a dotted line) exist almost solely in those regions where $\Delta h$ predicted by theory happens to be $\approx-\tilde{h}(\Delta h \approx-5 \approx-\tilde{h}$ in the case displayed in this Figure). This is not a coincidence, and what really happens is the following. First, planetesimals scatter and their orbital elements change in full agreement 

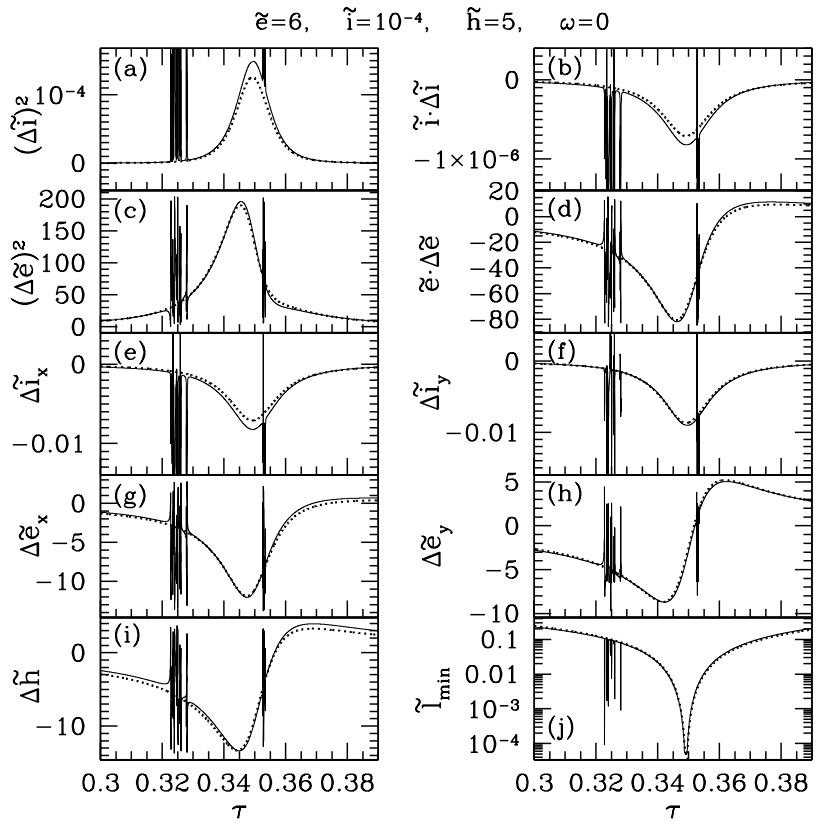

FIG. 8.- Changes of relative orbital parameters (a) $(\Delta \tilde{i})^{2}$, (b) $\tilde{i} \cdot \Delta \tilde{i}$, (c) $(\Delta \tilde{e})^{2}$, (d) $\tilde{e} \cdot \Delta \tilde{e}$, (e) $\Delta \tilde{i}_{x}$, (f) $\Delta \tilde{i}_{y}$, (g) $\Delta \tilde{e}_{x},(\mathrm{~h})$ $\Delta \tilde{e}_{y}$, (i) $\Delta \tilde{h}$, and (j) the minimum separation $\tilde{l}_{\min }$ of planetesimals plotted as a function of their relative horizontal epicyclic phase $\tau$ (at large separation prior to scattering). Initial orbital parameters corresponding to this calculation are shown at the top of the plot. Only an interval $0.3<\tau<0.39$ in which strong scattering takes place is displayed. Solid curves show the numerical results while the dotted lines are the analytical predictions. Note the chaotic variation of orbital parameters for $0.32<\tau<0.33$ and $0.35<\tau<$ 0.355 .

with analytical theory. This means, however, that postscattering $\tilde{h} \sim 1$ and the guiding center of the planetesimal orbit is now moving very slowly with respect to the scatterer, while eccentricity is still quite high. Right after scattering planetesimals are very close to each other, and Keplerian shear does not allow their guiding centers to recede very far because $\tilde{h}$ is small, so that after one orbital period planetesimals may closely approach each other again and experience another scattering. This second scattering may or may not dislodge them from close proximity of each other but it will certainly affect their final orbital elements, explaining the deviation of $\Delta \tilde{h}$ and other orbital elements from theoretical predictions when $\Delta h \approx-\tilde{h}$. Thus, we conclude that

- Chaotic variations of orbital elements result from multiple scatterings of planetesimals in the course of close encounter rather than from the failure of the two-body approximation.

- Phase intervals where theoretical $\Delta \tilde{h} \approx-\tilde{h}$ are naturally occupied by chaotic orbits (there are also other possibilities for producing multiple scattering orbits, see below).

These observations greatly help in explaining the puzzling results for the inclination-based scattering coefficients. From pure geometry it is clear that the highest inclination $\tilde{i}_{1}$ which a high-velocity particle with initial inclination $\tilde{i}_{0}$ can attain after a single scattering event is $^{6}$

$$
\tilde{i}_{1} \sim \tilde{i}_{0} \tilde{v}_{0}^{3} .
$$

This is easily seen in Figures $8 \mathrm{k}, \mathrm{f}$ which show that maximum $\tilde{i}_{1} \sim 10^{-2}$ for $\tilde{i}_{0} \sim 10^{-4}$ : since $\tilde{v}_{0}=\left(\tilde{e}^{2}-\right.$ $\left.(3 / 4) \tilde{h}^{2}\right)^{1 / 2} \approx 4.2$ one should expect maximum $\tilde{i}_{1} \sim$ $10^{-4} \times 4.2^{3} \approx 0.007$, very close to what we find in reality outside of the region of chaotic orbits.

At the same time Figures 8 , f show that chaotic orbits often exhibit final $\tilde{i}$ much larger than predicted by equation (39). This, of course, is naturally explained by the fact that chaotic orbits result from multiple scattering. Every scattering of a high-velocity orbit can potentially increase inclination by a factor $\tilde{v}_{0}^{3} \gg 1$ (the approach velocity of planetesimals does not change very strongly after multiple scatterings and is still $\sim \tilde{v}_{0}$; this can be understood from the conservation of Jacobi constant). Thus, after $n$ scatterings maximum possible inclination would have been $\tilde{i}_{n} \sim \tilde{i}_{0} \tilde{v}_{0}^{3 n}$, except that in practice $\tilde{i}$ cannot exceed $\tilde{v}_{0}$. The highest final $\tilde{i}$ that we could find for the parameters of Figure 8 is $\approx 0.7$ but one has to keep in mind that orbits in the chaotic region exhibit quasi-fractal behavior in that the denser is the grid in $\tau$ used for computing $\Delta \tilde{i}$ the richer the behavior found. Thus, we could have easily missed orbits with even higher final $\tilde{i}$. On theoretical grounds we expect the maximum $\tilde{i}$ in this Figure at the level of $10^{-4} \times 4.2^{3 \times 2} \approx 0.5$ if only $n=2$ close scatterings have taken place.

In Figure 9 we illustrate a multiple scattering event for an orbit with initial parameters $\tilde{e}=15, \tilde{i}=10^{-6}$ and $\tilde{h} \approx 12.6$ (in this case $\tilde{v}_{0} \approx 10.2$ ). From Figure $9 \mathrm{~b}, \mathrm{c}$ one can see that as a result of the first scattering $\tilde{h}$ becomes very small while $\tilde{i}$ jumps up by $\sim 10^{2}$. After that the planetesimal loops around its scatterer for several orbital periods, as illustrated in Figures $9 \mathrm{~d}-\mathrm{f}$, until the second strong scattering takes place, resulting in final $\tilde{h} \approx 20$. This allows the planetesimal to leave the vicinity of its scatterer. During the second scattering $\tilde{i}$ is again boosted up by more than two orders of magnitude, resulting in final $\tilde{i} \approx 0.03$. This is much larger than $10^{-6} \times 10.2^{3} \approx$ $10^{-3}$ - the maximum $\tilde{i}_{1}$ one would expect from single scattering.

It now becomes much easier to understand the erratic behavior of scattering coefficients $\hat{K}_{1,2}$ in Figure 2 In particular, multiple scattering orbits very strongly affect $\hat{K}_{1}$ since $\Delta \tilde{i}$ enters the calculation of this stirring coefficient in a second power. Because of that, even though chaotic orbits arise for only a small subset of horizontal epicyclic phases they affect the value of numerically determined $\hat{K}_{1}$ very strongly. According to equation (27) $\left\langle(\Delta \tilde{i})^{2}\right\rangle_{\omega, \tau} \approx 6.5 \times 10^{-10}$ for the values of $\tilde{e}, \tilde{h}$ and $\tilde{i}$ used in making Figure 9. At the same time a single orbit as displayed in Figure 9 has $(\Delta \tilde{i})^{2} \sim 10^{-4}$, more than $10^{6}$ times higher than the theoretical phase average of this

6 Highest inclination results from scattering by $\approx \pi / 2$, which requires impact parameter of incoming trajectory to be $\tilde{l} \sim \tilde{v}_{0}^{-2}$, at initial vertical separation of order $\tilde{i}_{0} R_{H}$. The final velocity of the receding planetesimal is $\tilde{v}_{0}$ and from simple geometry its vertical component (which is equivalent to inclination in Hill units) is $\tilde{v}_{0} \times\left(\tilde{i}_{0} / \tilde{l}\right) \sim \tilde{i}_{0} \tilde{v}_{0}^{3}$. 
$\tilde{\mathrm{h}} \approx 12.6, \gamma=3000, \tilde{\mathrm{e}}_{\mathrm{x}} \approx 8.5, \tilde{\mathrm{e}}_{\mathrm{y}} \approx 12.3, \tilde{\mathrm{i}}_{\mathrm{x}} \approx 4.1 \mathrm{e}-07, \tilde{\mathrm{i}}_{\mathrm{y}} \approx 9.1 \mathrm{e}-07$
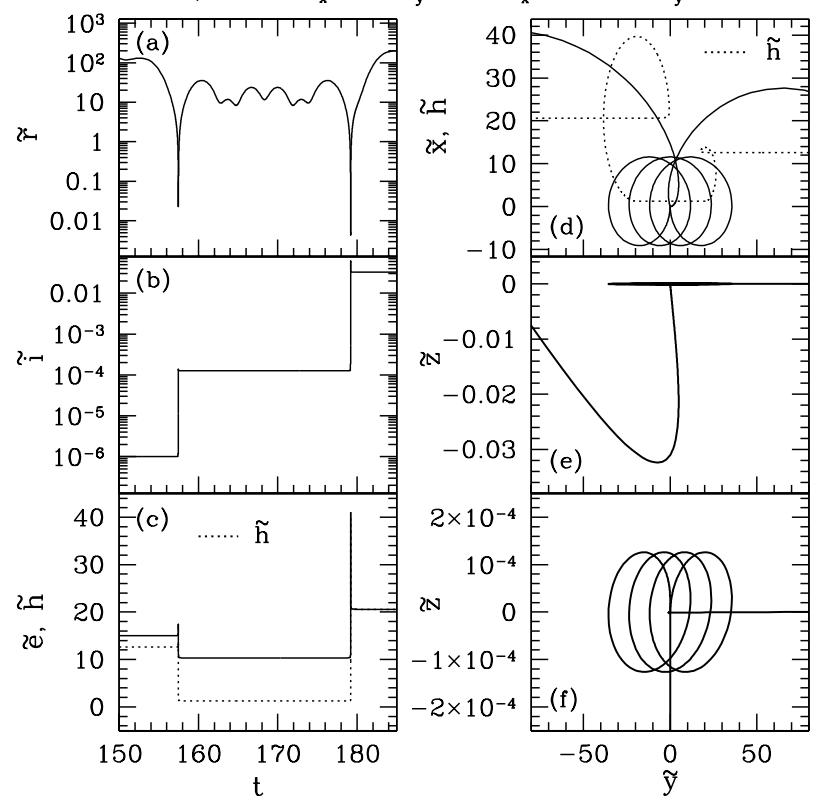

FIG. 9.- (a-c) Variation of the relative orbital elements of two planetesimals in the course of a multiple scattering event, for initial orbital elements indicated at the top of the plot. Evolution of (a) the relative distance between the bodies $\tilde{r},(\mathrm{~b})$ their relative inclination $\tilde{i}$, and (c) their $\tilde{e}$ (solid line) and $\tilde{h}$ (dotted line) are shown. This trajectory exhibits two strong scattering events in each of which $\tilde{i}$ gets boosted up by two orders of magnitude. (d-f) Trajectory of relative motion in the course of scattering shown (d) in the $\tilde{y}-\tilde{x}$ coordinates and (e) in $\tilde{y}-\tilde{z}$ coordinates. A zoomed in version of panel (e) is shown in panel (f) to better illustrate the complexity of vertical motion before the final scattering causes planetesimals to recede from each other. In panel (d) we show both the instantaneous position of planetesimal that is being scattered (solid line) and the trajectory of its guiding center (dotted line). See text for more details.

quantity. It is thus not surprising that even though we have used a very large number of orbits in computing scattering coefficients (according to the prescription (38) our calculation of $\hat{K}_{1}$ for $\tilde{e}=15$ used 13.3 million orbits) chaotic orbits still affect them quite significantly.

We can now explain why the scatter in numerical values of $\hat{K}_{1}$ and the deviation from analytical prediction both become stronger as $\tilde{i}$ decreases: the maximum possible value of $\tilde{i}$ resulting from scattering is always limited from above by $\tilde{i} \sim \tilde{v}_{0}$, so that the maximum stochastic $(\Delta \tilde{i})^{2} \sim v_{0}^{2}$, independent of initial $\tilde{i}$. However, the analytical value of $\hat{K}_{1} \propto \tilde{i}^{2}$, so that the ratio of analytical $\hat{K}_{1}$ to the numerical one increases as $\tilde{i}$ decreases.

It is not even clear that our calculation of $\hat{K}_{1}$ in Figure 2 has converged - one cannot guarantee that increasing the number of orbits would not increase even more the number of extremely chaotic orbits with very large $\Delta \tilde{i}$, which would then dominate the calculation. The only thing that argues against this scenario is the saturation of final $\tilde{i}$ at the level of $\tilde{v}_{0}$ even for very large number of repeated scatterings. Nevertheless, until we understand how much of the phase space volume corresponds to chaotic orbits with very large $\Delta \tilde{i}$ we cannot draw a final conclusion about the convergence of $\hat{K}_{1}$ and we leave

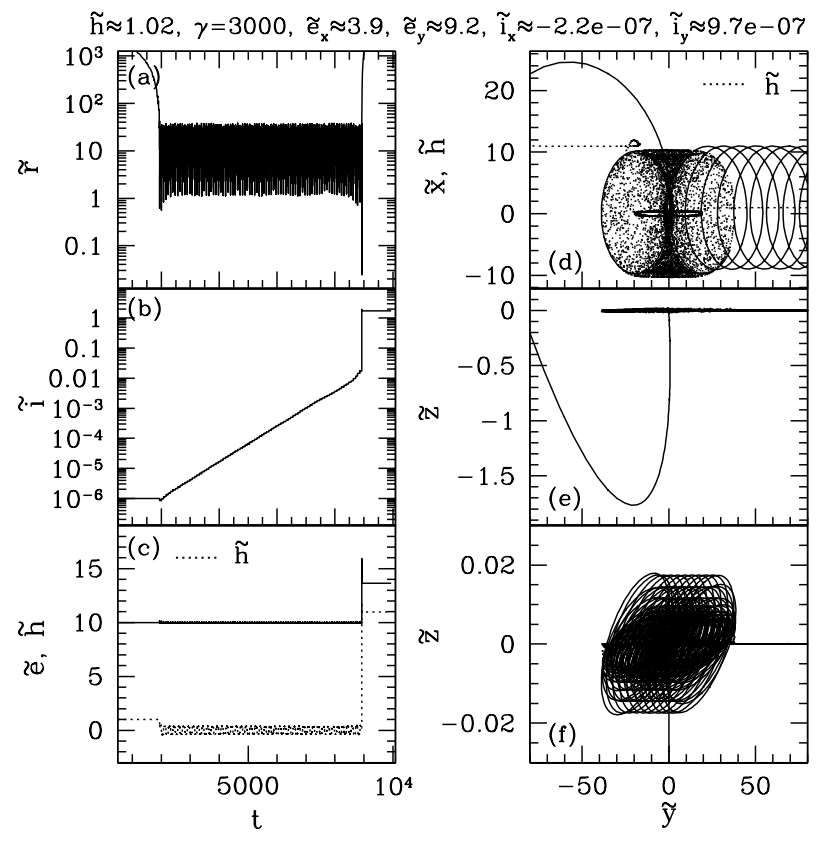

FIG. 10. - Same as Figure 9 but for a different choice of orbital elements indicated at the top of the plot characterized by small initial $\tilde{h}$. Note an exponential growth of $\tilde{i}$ by several orders of magnitude in panel (b). In panel (d) we plot only a small fraction of data during the scattering event as dots to better illustrate the underlying structure. See text for more details.

this subject for future investigation. Paradoxically, the agreement between the numerical and analytical results may be better if one uses smaller number of orbits in numerical calculation of $\hat{K}_{1,2}$ since then the chance of randomly picking one of the high- $\Delta \tilde{i}$, multiple scattering orbits is also smaller.

In the course of our investigation we have also found that orbits with $\tilde{e} \gg 1$ and $\tilde{h} \gg 1$ (like the one shown in Figure 9) are not the biggest contributors to chaos in $\hat{K}_{1,2}$. It turns out that inclination-based scattering coefficients are most strongly affected by orbits with $\tilde{e} \gg 1$ and $\tilde{h} \sim 1$, i.e. orbits which are initially close to the separatrix between the horseshoe and passing orbits. An example of planetesimal scattering corresponding to this case is shown in Figure 10 for initial $\tilde{h} \approx 1.02$, $\tilde{e}=10, \tilde{i}=10^{-6}$. This event is characterized by a very long time interval, more than $10^{3}$ orbital periods, during which planetesimals stay close to each other. They essentially form a temporary distant satellite system (note that the distance between planetesimals is larger than $R_{H}$ ), which slowly evolves in time. Figure 9b demonstrates that during this temporary capture $\tilde{h}$ oscillates around zero not allowing planetesimals ro recede from each other. Their relative inclination increases exponentially (with rather long time constant) by 4 orders of magnitude in an orderly fashion. Finally a strong scattering event occurs, which boosts up $\tilde{i}$ by $\sim 10^{2}$ and dislodges the planetesimal from its scatterer's vicinity. For this event $(\Delta \tilde{i})^{2} \sim 1$, while a single scattering calculation would predict $\left\langle(\Delta \tilde{i})^{2}\right\rangle_{\omega, \tau} \approx 6.7 \times 10^{-9}$ for the values of $\tilde{e}, \tilde{h}$ and $\tilde{i}$ shown on top of Figure 11. As a 

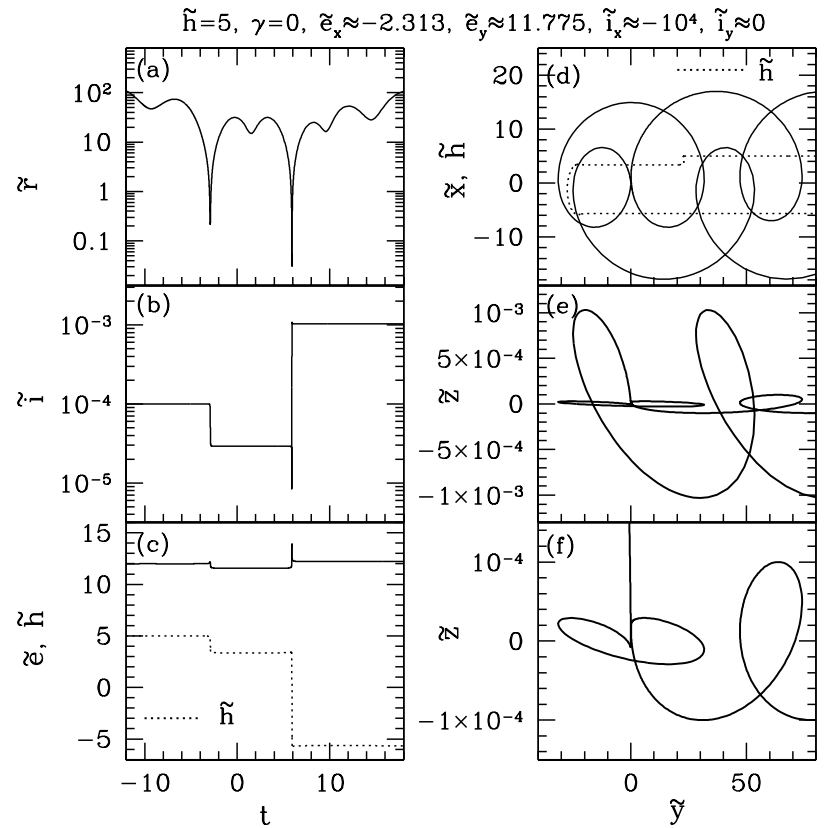

FIG. 11. - Same as Figure 10 but for a different choice of orbital elements indicated at the top of the plot. Note that while after the first scattering $\tilde{h}$ is not particularly close to unity, multiple scattering still takes place. See text for more details.

result, this single scattering event completely determines the calculation of $\hat{K}_{1}$.

It is worth pointing out here that multiple scattering in general does not require $\Delta \tilde{h} \approx-\tilde{h}$ and Figure [1] illustrates this statement. This Figure shows a double scattering event for initial $\tilde{h}=5, \tilde{e}=12$, and $\tilde{i}=10^{-4}$. As can be seen in Figure 11k after the first strong scattering event $\tilde{h} \approx 3.5$ and Keplerian shear ensures that the bodies will not stay close to each other for very long. However, before the planetesimal leaves its scatterer's vicinity its epicyclic motion brings it back into their mutual Hill sphere where another scattering event occurs. This type of multiple scattering event does not affect coefficients $\hat{K}_{1,2}$ nearly as much as events with $\Delta \tilde{h} \approx-\tilde{h}$.

To summarize, multiple scattering explains the discrepancy between the analytical and numerical results for $\hat{K}_{1}$ and stochastic scatter at high $\tilde{e}$ in values of $\hat{K}_{2}$ quite well. However, this explanation does not work so well for the systematic deviation of the numerical $\hat{K}_{2}$ from the analytical one clearly seen in Figure $2 \mathrm{~d}$ for virtually all values of $\tilde{e}$ : even at small $\tilde{e}$, when the stochastic scatter is small, $\hat{K}_{2}$ increases contrary to theory. Note that while the lower envelope of $\hat{K}_{1}$ for a given $\tilde{i}$ agrees quite well with the analytical prediction (and multiple scattering explains the remaining stochasticity), this is clearly not true for $\hat{K}_{2}$.

\subsection{Distant interaction.}

To understand the systematic deviation of the numerical $\hat{K}_{2}$ from the analytical prediction (32) we first note that coefficient $\langle\tilde{\mathbf{i}} \cdot \Delta \tilde{\mathbf{i}}\rangle_{\omega, \tau}$ used in calculating of $\hat{K}_{2}$ also systematically differs from the analytical prediction given by equation (28); see Figures $6 \mathrm{~d}, 7 \mathrm{~d}$. This may seem
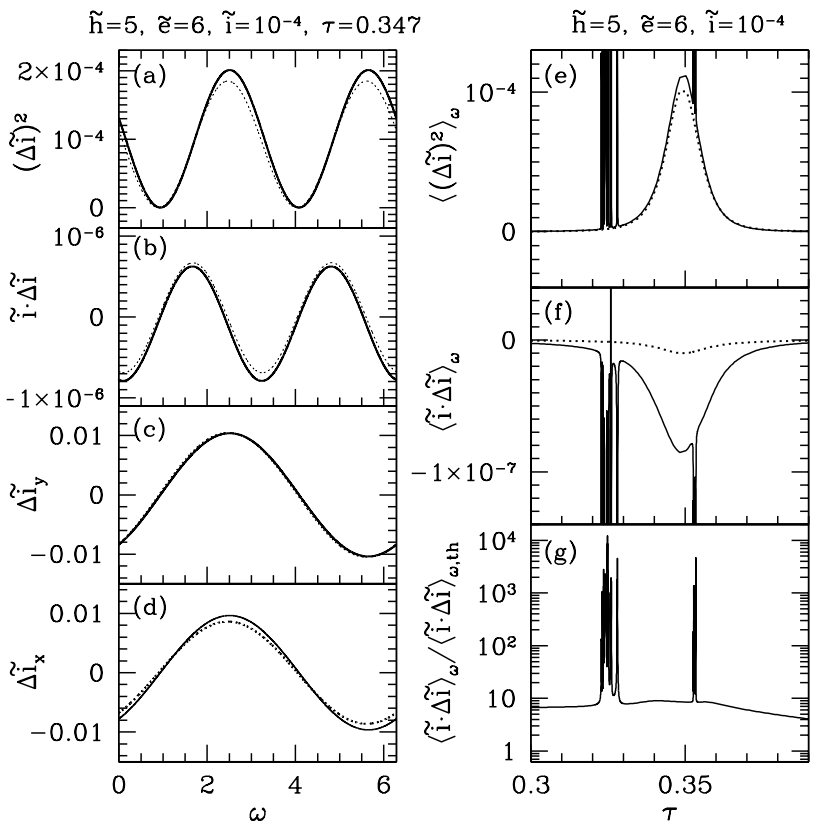

FIG. 12.- (a-d) Variation of (a) $(\Delta \tilde{i})^{2}$, (b) $\tilde{i} \cdot \Delta \tilde{i}$, (c) $\Delta \tilde{i}_{y}$, and (d) $\Delta \tilde{i}_{x}$ with vertical epicyclic phase $\omega$ for a particular set of initial orbital parameters (shown on top of left column). Theoretical predictions are shown with the dotted line while numerical results are in solid. (e-g) Plots of (e) $(\Delta \tilde{i})^{2}$ and (f) $\tilde{i} \cdot \Delta \tilde{i}$ averaged over $\omega$ as functions of $\tau$ for a particular set of initial orbital parameters (shown on top of right column). Solid lines are numerical results, dotted lines are analytical predictions. Panel $(\mathrm{g})$ shows the ratio of the numerically computed average of $\tilde{i} \cdot \Delta \tilde{i}$ over $\omega$ to the analytical prediction for the same quantity. Panels (f) and (g) clearly demonstrate that theory underpredicts $\langle\tilde{i} \cdot \Delta \tilde{i}\rangle_{\omega}$ by about an order of magnitude.

surprising since in Figure $8 \mathrm{~b}$ the numerically determined $\tilde{\mathbf{i}} \cdot \Delta \tilde{\mathbf{i}}$ follows quite closely analytical prediction as a function of horizontal phase $\tau$. Chaotic variations of $\tilde{\mathbf{i}} \cdot \Delta \tilde{\mathbf{i}}$ due to multiple scattering noticeable in this plot within narrow intervals of $\tau$, cannot explain the systematic discrepancy for $\hat{K}_{2}$ - they can only be responsible for the random scatter in the calculation of $\hat{K}_{2}$. However, Figure $8 \mathrm{~b}$ does not show how $\tilde{\mathbf{i}} \cdot \Delta \tilde{\mathbf{i}}$ depends on $\omega$ (this Figure was made for a single value of $\omega$ ) and this dependence turns out to be very important.

In Figure 12 a-d we display the dependence of $(\Delta \tilde{i})^{2}$, $\tilde{i} \cdot \Delta \tilde{i}, \Delta \tilde{i}_{y}$, and $\Delta \tilde{i}_{x}$ on $\omega$ for a fixed $\tau$. The value of $\tau=0.347$ is chosen in order to avoid intervals of chaotic variation of the orbital elements ${ }^{7}$ in order to isolate the subsequent analysis from the effects of multiple scattering. The general agreement of the numerical and analytical curves, including those of $\tilde{i} \cdot \Delta \tilde{i}$, is quite good in this Figure. However, to calculate $\langle\tilde{\mathbf{i}} \cdot \Delta \tilde{\mathbf{i}}\rangle_{\omega, \tau}$ we need to integrate $\tilde{i} \cdot \Delta \tilde{i}$ over $\omega$ and it is quite obvious from Figure 12 b that $\tilde{i} \cdot \Delta \tilde{i}$ is very close to a pure sinusoid. Its $\omega$ average $\langle\tilde{\mathbf{i}} \cdot \Delta \tilde{\mathbf{i}}\rangle_{\omega}$ should then be very small and strongly dependent on the deviations of $\tilde{i} \cdot \Delta \tilde{i}$ from a pure sinusoid. As a result, if the numerical and analytical values

7 See Figure 8 which is made for the same set of initial orbital parameters as Figure 12 
of $\tilde{i} \cdot \Delta \tilde{i}$ deviate from the sinusoid differently, one can get a significant discrepancy between theory and numerical calculation.

This is exactly what is going on as we demonstrate in Figure 12 , f where we plot $\left\langle(\Delta \tilde{i})^{2}\right\rangle_{\omega}$ and $\langle\tilde{\mathbf{i}} \cdot \Delta \tilde{\mathbf{i}}\rangle_{\omega}$ as functions of $\tau$. One can see that averaging over $\omega$ does not affect the agreement between numerical and analytical $(\Delta \tilde{i})^{2}$ seen in Figure $12 \mathrm{a}$ because it is an intrinsically positive quantity. But $\omega$-averaging of $\tilde{i} \cdot \Delta \tilde{i}$ does lead to a dramatic difference between analytical and numerical $\langle\tilde{\mathbf{i}} \cdot \Delta \tilde{\mathbf{i}}\rangle_{\omega}$ in Figure 12f for the reason we just described. In Figure 12 , we show the ratio of $\langle\tilde{\mathbf{i}} \cdot \Delta \tilde{\mathbf{i}}\rangle_{\omega}$ determined by the two methods, and one can clearly see that the numerical result significantly exceeds the analytical one (both in regions of chaotic and orderly behavior of orbital parameters), in agreement with the fact that the numerical $\hat{K}_{2}$ is systematically higher than the analytical $\hat{K}_{2}$, see Figure 2d.

What may produce such a difference in scaling of analytical and numerical $\tilde{i} \cdot \Delta \tilde{i}$ with $\omega$ ? In Appendix B we provide a simple calculation of $\langle\tilde{\mathbf{i}} \cdot \Delta \tilde{\mathbf{i}}\rangle_{\omega}$ allowing for a small but non-zero difference $\delta \omega_{\text {dist }}$ of the relative vertical epicyclic phase of interacting planetesimals $\omega$ at large separation and right before the close encounter. Such a phase difference in $\omega$ arises because of the distant interaction between planetesimals prior to their encounter and is analogous to the shift in horizontal phase $\tau$ which was invoked in Figure 8 to better match analytical and numerical results (see also Tanaka \& Ida 1996). We show in Appendix B that although $\left|\delta \omega_{\text {dist }}\right|$ is expected to be small its effect on the calculation of $\langle\tilde{\mathbf{i}} \cdot \Delta \tilde{\mathbf{i}}\rangle_{\omega}$ is very important (and dominates this calculation) as long as $\tilde{v}_{0}^{3}\left|\delta \omega_{\text {dist }}\right| \gtrsim 1$.

The rather surprising result that a small variation of vertical phase $\omega$ can strongly affect the calculation of dynamical friction coefficients $\hat{K}_{2}$ and $K_{2}$ is explained by strong cancellation that takes place when one averages $\tilde{\mathbf{i}} \cdot \Delta \tilde{\mathbf{i}}$ over $\omega$ : some terms proportional to $\delta \omega_{\text {dist }}$ that average to zero when $\delta \omega_{\text {dist }} \equiv 0$ can become very large after averaging when $\delta \omega_{\text {dist }} \neq 0$. In graphical form the same issue has already been illustrated in Figure 12. Also, a careful inspection of our calculation of all other scattering coefficients including $\left\langle(\Delta \tilde{i})^{2}\right\rangle_{\omega, \tau}$ shows that unlike $\langle\tilde{\mathbf{i}} \cdot \Delta \tilde{\mathbf{i}}\rangle_{\omega, \tau}$ they are not affected by non-zero $\delta \omega_{\text {dist }} \neq 0$ since they do not suffer from cancellation effects when averaged over $\omega$. For these coefficients, our calculation neglecting the effects of distant interaction presented in Appendix $\mathrm{A}$ remains valid.

Another important point to make here is that although there is a large difference between the numerical and analytical $\hat{K}_{2}$ and $K_{2}$, this discrepancy is not always critical for the inclination evolution of planetesimals in the regime (24). Indeed, as Figures [45] show, $K_{1} / K_{2} \sim 10^{4}$ for $\tilde{\sigma}_{e} \gtrsim 5-10$, which according to equation (18) implies that the gravitational friction term becomes important for inclination evolution only if $\mu_{1} \gg \mu_{2}$, i.e. for the velocity evolution of massive bodies driven by their interaction with low-mass planetesimals.

The issue of distant interaction and its effect on the scattering calculation clearly deserves further work but we postpone it for a separate investigation. For now it is enough for us to just state that distant interactions serve as a plausible explanation for the deviations between the numerical and analytical calculations of $\langle\tilde{\mathbf{i}} \cdot \Delta \tilde{\mathbf{i}}\rangle_{\omega, \tau}, \hat{K}_{2}$ and $K_{2}$.

\section{VELOCITY EVOLUTION OF PROTOPLANETARY CORES.}

Here we use our results as obtained in previous sections to understand the velocity evolution of a sparse population of protoplanetary cores in the end of oligarchic phase, when their orbits become crossing - a situation described in $\$ 1$ For simplicity we will assume the masses of all cores $M_{c}$ to be roughly equal, i.e. $\mu_{1}=\mu_{2}=\mu=M_{c} / M_{\star}$ and $\sigma_{\{e, i\}, 1}=\sigma_{\{e, i\}, 2}=\sigma_{\{e, i\}}$, which allows us to omit the term proportional to $K_{2}$ in the equation for inclination evolution; see 44.4 Also, we will neglect the effects of multiple scattering on the inclination evolution, which is justified to some extent by the small number of bodies involved (and the small number of their close encounters); see \$44.3. We can then use our analytical expressions for the scattering coefficients to study velocity evolution.

We assume that initially $\tilde{\sigma}_{e}=\tilde{\sigma}_{e 0} \gg 1$ and $\tilde{\sigma}_{i}=\tilde{\sigma}_{i 0}$ satisfies constraint (24). Equation (18) supplemented with expressions for the scattering coefficients yields the following set of velocity evolution equations:

$$
\begin{aligned}
\frac{d \sigma_{e}}{d t} & =C_{e} T_{e}^{-1} \mu^{1 / 3}, \\
\frac{d \sigma_{i}}{d t} & =C_{i} T_{e}^{-1} \sigma_{i} \tilde{\sigma}_{e}^{5},
\end{aligned}
$$

where evolution time $T_{e}$ is given by

$$
\begin{aligned}
& T_{e}=\frac{1}{\Omega N_{p} R_{H}^{2}}=\frac{\mu^{1 / 3} M_{\star}}{\Omega \Sigma_{p} a^{2}} \\
& \approx 150 \mathrm{yr}\left(\frac{M_{c}}{0.01 M_{\oplus}}\right)^{1 / 3}\left(\frac{\mathrm{AU}}{a}\right)^{1 / 2}\left(\frac{30 \mathrm{~g} \mathrm{~cm}^{-2}}{\Sigma_{p}}\right)
\end{aligned}
$$

for $M_{\star}=M_{\odot}$, and coefficients $C_{e}, C_{i}$ are constants of order unity (their values can be found from equations (33) - (35)). Note that these equations apply equally well both to the dense population of planetesimals with overlapping orbits and to the sparse population of cores with crossing orbits.

One can understand the origin of these equations qualitatively based on the fact that scattering coefficients are dominated by the large-angle scattering events in the thin-disk case, i.e. those that require impact parameter $l_{\min } \sim R_{H} / \tilde{v}_{0}^{2}$. Since we consider the case $\tilde{e} \gg 1$ the relative velocity between planetesimals is $\sim \tilde{v}_{0} \Omega R_{H}$. Then a mean time between encounters of a given body with other bodies at an impact parameter $l_{\text {min }}$ is $T_{\pi / 2} \sim \tilde{v}_{0} /\left(\Omega N_{p} R_{H}^{2}\right)$, where $N_{p}=\Sigma_{p} / M_{c}$ is the planetesimal (or core) surface number density. When a large-angle scattering event occurs $e^{2}$ changes by $\sim e^{2}$, while $i^{2}$ changes by $\sim i^{2} \tilde{v}_{0}^{6}$, see $\$ 44.3$. Then $d \sigma_{e}^{2} / d t \sim e^{2} / T_{\pi / 2}$, while $d \sigma_{i}^{2} / d t \sim i^{2} \tilde{v}_{0}^{6} / T_{\pi / 2}$, which results in equations (40)-(41) if we recall that $\tilde{v}_{0} \sim \tilde{\sigma}_{e}$.

Integrating equations (40)-(41) we find

$$
\begin{aligned}
& \tilde{\sigma}_{e}=\tilde{\sigma}_{e 0}+C_{e} \frac{t}{T_{e}} \\
& \tilde{\sigma}_{i}=\tilde{\sigma}_{i 0} \exp \left[\frac{C_{i}}{6 C_{e}}\left(\tilde{\sigma}_{e}^{6}-\tilde{\sigma}_{e 0}^{6}\right)\right] .
\end{aligned}
$$


For $t \ll T_{e}$ one has $\tilde{\sigma}_{e} \approx \tilde{\sigma}_{e 0}$ and

$$
\tilde{\sigma}_{i}=\tilde{\sigma}_{i 0} \exp \left[C_{i} \tilde{\sigma}_{e 0}^{5} \frac{t}{T_{e}}\right] .
$$

One can see from these solutions that growth of $\sigma_{i}$ has a rather explosive character: $\sigma_{i}$ increases exponentially and at the very beginning the inclination growth timescale is $\sim \tilde{\sigma}_{e 0}^{-5} T_{e} \ll T_{e}$ since $\sigma_{e 0} \gtrsim 1$. As a result, while $\sigma_{i}$ grows by several orders of magnitude, $\sigma_{e}$ does not change that much. This means that protoplanetary cores should very rapidly transition from the very thin, almost 2D configuration to a vertically extended disk in which the condition (24) is no longer fulfilled.

In reality inclination will grow not in a continuous fashion as described by equations (44) and (45) but more in a step-like way by a factor of $\sim \tilde{v}_{0}^{3}$ as large-angle scattering events occur at time intervals of order $T_{\pi / 2}$. Continuous description is going to be useful only after a large number of large-angle scattering events have taken place (and during this period $\tilde{i}$ would have grown a lot).

Onset of the core orbit crossings not only increases the cores' inclination but also makes possible collisions between cores leading to their growth. While the disk is geometrically thin, the collision probability of cores is rather large, and one may wonder whether the cores would grow appreciably during the short period of time while the condition (24) is still fulfilled. What matters for the core growth during this period is both the accretion rate (which is very high) and the time during which the inclination of the disk is still in the regime (24), which is short. Careful analysis shows that the relative core mass increase during the "thin disk" epoch of the core population evolution is very small, much less than unity, provided that $\tilde{v}_{0}$ is less than the escape speed from the core surface. A simple reason for this is that when the relative speed of bodies is less than their surface escape speed $l_{\text {min }}$ is larger than the impact parameter leading to collisions between the cores, so that the inclination strongly increases before cores have had a significant chance to collide. Thus, all of the late core agglomeration resulting in present day terrestrial planets occurs only after the disk has become geometrically rather thick, i.e. already when $\sigma_{i} \sim \sigma_{e}$.

A similar picture of velocity evolution - relatively rapid growth of inclination compared to the growth of eccentricity - is expected also in the rather general situation of a planetesimal disk transitioning from the shear-dominated to the dispersion-dominated dynamical regime as a result of gravitational scattering. Indeed, in the shear-dominated case eccentricity growth is expected to be much faster than the growth of inclination because of the geometry of gravitational scattering of planetesimals in a dynamically cold disk. Thus, when $\tilde{\sigma}_{e}$ becomes comparable to unity and continues to grow the condition (24) is fulfilled, meaning that very soon after leaving the shear-dominated regime the planetesimal disk should rapidly increase its inclination in accordance with equations (43)-(45). This qualitative picture has indeed been observed in calculations of planetesimal velocity evolution based on direct N-body simulations (Ida \& Makino 1992).

6. DISCUSSION.
In this paper we have explored for the first time a rather special dynamical regime of planetesimal velocity evolution represented by the condition (24). Previous work towards understanding planetesimal dynamics has been primarily focused on thick planetesimal disks with $i \sim e$ (e.g. Stewart \& Ida 2000; Ohtsuki et al. 2002). Thin disks have been considered by Palmer et al. (1993) but their study assumed a razor-thin disk, i.e. $i=0$, which precluded them from studying a very important aspect of the problem - excitation of inclination in a thin disk. They have explored horizontal velocity excitation in a 2D disk, however, their results cannot be directly compared with ours: Palmer et al. computed quantities like $d v_{r}^{2} / d t-$ growth rate of the radial velocity dispersion of planetesimals - which cannot be directly related to our $d \sigma_{e}^{2} / d t$ since for the latter one also needs to know the growth rate of azimuthal velocity dispersion. Nevertheless, their $d v_{r}^{2} / d t$ scales linearly with $\sigma_{e}$, in agreement with our equations (29), (30) and (33), (34) for eccentricity-based scattering coefficients. Ida (1990) has also recovered a linear dependence of $2 \mathrm{D}$ horizontal excitation rates on $\sigma_{e}$ numerically.

The transition between the thin-disk and thick-disk regimes of scattering which occurs at $\tilde{i} \sim \tilde{i}_{\text {crit }}$ has not been previously investigated. It is known from the studies of thick planetesimal disks that scattering coefficients tend to diverge as $\tilde{i} \rightarrow 0$ (e.g. Stewart \& Ida 2000). On the other hand, Ida (1990) has found numerically that in a purely $2 \mathrm{D}$ disk horizontal scattering coefficients are finite, which led him to a conjecture that these coefficients should change discontinuously at $\tilde{i}=0$. Our present study shows this not to be the case. Instead, 3D rates increase with decreasing $\tilde{i}$ until $\tilde{i}$ reaches $\tilde{i}_{\text {crit }}$ at which point the geometry of scattering changes and scattering coefficients smoothly transition to their $2 \mathrm{D}$ values. This process is best illustrated in Figure 3 where we plot both our thin disk results and the scaling of scattering coefficients with $\tilde{i}$ in the $3 \mathrm{D}$ regime. Using analytical expressions for various scattering coefficients in Stewart \& Ida (2000) we have verified that the magnitudes of 3D scattering coefficients coincide (up to numerical constant of order unity) with values of our $2 \mathrm{D}$ scattering coefficients at $\tilde{i} \sim \tilde{i}_{\text {crit }}$.

Our final comment concerns multiple scattering encounters resulting in temporary captures such as the orbit shown in Figure 10. A long time spent by one body in the vicinity of the Hill sphere of another opens up the possibility of capturing this body into a distant satellite orbit if some weak additional perturbation (e.g. gas drag, collision with/or gravitational perturbation by an additional passing planetesimal) affects the mutual orbit of the bodies. By distant satellite we imply a satellite with separation larger than the mutual Hill sphere of the two bodies, and it seems plausible that the formation of such a configuration should somehow involve a high velocity encounter between the two objects (relative speed of a distant satellite and its parent body exceeds Hill velocity $\left.\Omega R_{H}\right)$.

Multiple scattering encounters between low-velocity planetesimals potentially leading to the formation of satellites with separations less than $R_{H}$ have been studied by e.g. Iwasaki \& Ohtsuki (2007) and Schlichting \& Sari (2008). The high-velocity regime of multiple scat- 
tering and temporary capture has not yet been explored theoretically and is likely to differ from the low-velocity regime. In particular, Schlichting \& Sari (2008) found that the probability of forming temporary satellite system drops exponentially with the duration of the temporary capture in the low-velocity regime, and that essentially no temporary capture systems should exist for more than several tens of $\Omega^{-1}$. However, in the highvelocity case we are finding orbits like the one displayed in Figure 10. which exhibit temporary capture for more than $10^{3} \Omega^{-1}$.

Distant satellites have not yet been discovered in planetary systems but their dynamics were investigated theoretically by a number of authors (Jackson 1913; Lidov \& Vashkov'yak 1994a,b). In particular, recently Shen \& Tremaine (2008) have demonstrated using a mapping approach that distant satellites around some planets (Jupiter, Uranus, and Neptune) are stable on time scales comparable to the life time of the Solar System. Temporary captures resulting from multiple scattering of dispersion-dominated planetesimals described in 44.3 present one of the possible ways in which such distant satellites may be formed. The dependence of the efficiency of this formation channel on the dynamical state of the planetesimal disk may provide us with an important probe of the dynamical characteristics of the early Solar System. Needless to say, issues like planetary migration or possible chaotic epochs of the dynamical evolution of the Solar System planets (Tsiganis et al. 2005; Gomes et al. 2005) must significantly complicate the interpretation of future detection (or non-detection) of distant satellites. Nevertheless, the investigation of their formation efficiency in temporary capture events like the one shown in Figure 10 is a worthwhile exercise.

\section{SUMMARY.}

We investigated the dynamical evolution of vertically thin, dispersion-dominated planetesimal disks with eccentricities and inclinations obeying the constraint (24).
In this regime of orbital parameters planetesimals see an anisotropic flux of incoming bodies (unlike in the case of thick disks), which dramatically changes the character of gravitational scattering. In particular, planetesimal velocity evolution is dominated by large-angle scattering events, unlike in the thick disk case. We derived analytical expressions for the scattering coefficients in the thin disk regime and compared them with numerical integrations of test orbits in the Hill approximation. We found good agreement between the two approaches for the eccentricity scattering coefficients, while the numerical inclination scattering coefficients significantly differ from their analytical analogs. We demonstrated that this discrepancy is caused by the important role of multiple scattering events not captured in our analytical calculations, and by the distant interactions of planetesimals in their approach phase before close encounter. Based on these results we have studied the velocity evolution of a population of protoplanetary cores in the end of the oligarchic phase and shown that the initially small inclination of this population grows very rapidly (exponentially) on a very short timescale. The results of this work are useful for understanding the velocity evolution of shear-dominated planetesimal disks at the transition to the dispersion-dominated regime and for the formation of distant satellites of planets.

We are grateful to Hilke Schlichting for careful reading of the manuscript and useful discussions. Financial support of this work was provided by the Sloan Foundation and NASA grant NNX08AH87G. ZS thanks the Advisory Council of the Department of Astrophysical Sciences, Princeton University, for generous support through a summer student fellowship. RR acknowledges the hospitality of Lebedev Physical Institute and Institute of Space Research during the completion stages of this work.

REFERENCES

Goldreich, P., Lithwick, Y., \& Sari, R. 2004, ARA\&A, 42, 549

Gomes, R., Levison, H. F., Tsiganis, K., \& Morbidelli, A. 2005, Nature, 435, 466

Gradshteyn, I.S. \& Ryzhik, I.M. Table of integrals, series, and products, New York: Academic Press, 1980.

Hasegawa, M. \& Nakazawa, K. 1990, A\&A, 227, 619

Hénon, M. \& Petit, J.M. 1986, Celestial Mechanics, 38, 67

Hornung, P., Pellat, R., \& Barge, P. 1985, Icarus, 64, 295

Ida, S. 1990, Icarus, 88, 129

Ida, S., Kokubo, E., \& Makino, J. 1993, MNRAS, 263, 875

Ida, S., \& Makino, J. 1992, Icarus, 96, 107

Ida, S., \& Makino, J. 1993, Icarus, 106, 210

Iwasaki, K. \& Ohtsuki, K. 2007, MNRAS, 377, 1763

Jackson, J. 1913, MNRAS, 74, 62

Kenyon, S.J. \& Bromley, B. C. 2004, AJ, 128, 1916

Kenyon, S.J. \& Bromley, B. C. 2009, ApJL, 690, L140

Kenyon, S.J. \& Luu, J.X. 1998, AJ, 115, 2136

Kokubo, E. \& Ida, S. 1998, Icarus, 131, 171

Lidov, M. L. \& Vashkov'yak, M. A. 1994a, Astron. Lett., 20, 188

Lidov, M. L. \& Vashkov'yak, M. A. 1994b, Astron. Lett., 20, 676

Ohtsuki, K., Stewart, G. R. \& Ida, S. 2002, Icarus, 155, 436
Palmer, P. L., Lin D. N. C., \& Aarseth, S. J. 1993, ApJ, 403, 336

Press, W. H., Flannery, B. P., Teukolsky, S. A., \& Vetterling, W. T. 1992, Numerical Recipes in C: The Art of Scientific Computing, Cambridge University Press; 2nd edition

Rafikov, R. R. 2003, AJ, 126, 2529

Rafikov, R. R. 2004, AJ, 128, 1348

Safronov, V.S. 1972, Evolution of the Protoplanetary Cloud and Formation of the Earth and Planets, NASA TT-F-677

Schlichting, H. E. \& Sari, R. 2008, ApJ, 673, 1218

Shen, Y. \& Tremaine, S. 2008, AJ, 136, 2453

Stewart, G. \& Ida, S. 2000, Icarus, 143, 28

Tanaka, H., \& Ida, S. 1996, Icarus, 120, 371

Tanaka, H., \& Ida, S. 1997, Icarus, 125, 302

Tsiganis, K., Gomes, R., Morbidelli, A., \& Levison, H. F. 2005, Nature, 435, 459

Wetherill, G. W. \& Stewart, G. R. 1989, Icarus, 77, 330

Wetherill, G. W. \& Stewart, G. R. 1993, Icarus, 106, 190

Youdin, A. 2008, astro-ph/0807.1114 


\section{APPENDIX}

\section{SCATTERING COEFFICIENTS IN THE 2D REGIME.}

To compute and analyze scattering coefficients characteristic for thin planetesimal disks we utilize an approach developed in Nakazawa et al. (1989), Ida et al. (1993), Tanaka \& Ida (1996). For given $\tilde{h}, \tilde{e}$, and $\tilde{i}$ there are two values of the horizontal phase $\tau_{c, \pm}$ and time $t_{c, \pm}$

$$
\tau_{c}^{ \pm}= \pm\left[\frac{4}{3}\left(\frac{\tilde{e}^{2}}{\tilde{h}^{2}}-1\right)^{1 / 2}-|\arccos (\tilde{h} / \tilde{e})|\right], \quad t_{c}^{ \pm}= \pm \frac{4}{3}\left(\frac{\tilde{e}^{2}}{\tilde{h}^{2}}-1\right)^{1 / 2},
$$

corresponding to the relative orbit passing through the origin (i.e. $x=y=0$ ) in the zero-inclination case (i.e. $z$ identically equal to zero). In the case of $\tilde{i} \sim \tilde{e}$, passage through the origin also implies that $z=0$ resulting in a constraint on $\omega$. However, in the case of a very thin disk with very small but non-zero inclination all values of $\omega$ correspond to roughly the same separation from the origin, which is mainly determined by the value of $\tau$. Orbits passing close to the origin can be expanded about $\tau_{c}^{ \pm}$in terms of $\eta^{ \pm}=\tau-\tau_{c}^{ \pm}$with the result that prior to interaction one planetesimal approaches another with velocity (scaled in Hill units by $\left.\Omega R_{H}\right) \tilde{\mathbf{v}}_{0}=\left(\tilde{v}_{0, x}, \tilde{v}_{0, y}, \tilde{v}_{0, z}\right)$ given by

$$
\tilde{v}_{0, x}^{ \pm}= \pm\left(\tilde{e}^{2}-\tilde{h}^{2}\right)^{1 / 2}, \quad \tilde{v}_{0, y}^{ \pm}=\frac{1}{2} \tilde{h}, \quad \tilde{v}_{0, z}^{ \pm}=\tilde{i} \cos \left(t_{c}^{ \pm}-\omega\right),
$$

and moving on a straight line orbit with the following coordinates of the point of closest approach:

$$
\tilde{x}_{c}^{ \pm}= \pm\left(\tilde{e}^{2}-\tilde{h}^{2}\right)^{1 / 2}\left(\frac{\tilde{e}^{2}}{\tilde{v}_{0}^{2}}-1\right) \eta^{ \pm}, \quad \tilde{y}_{c}^{ \pm}=\frac{1}{2} \tilde{h}\left(\frac{\tilde{e}^{2}}{\tilde{v}_{0}^{2}}-4\right) \eta^{ \pm}, \quad \tilde{z}_{c}^{ \pm}=\tilde{i} \sin \left(t_{c}^{ \pm}-\omega\right),
$$

where $\tilde{v}_{0}=\left|\tilde{\mathbf{v}}_{0}\right|=\left[\tilde{e}^{2}-(3 / 4) \tilde{h}^{2}\right]^{1 / 2}$. Impact parameter of the approach trajectory scaled by $R_{H}$ is

$$
\tilde{l}^{ \pm}=\frac{l^{ \pm}}{R_{H}}=\left[\left(\tilde{x}_{c}^{ \pm}\right)^{2}+\left(\tilde{y}_{c}^{ \pm}\right)^{2}+\left(\tilde{z}_{c}^{ \pm}\right)^{2}\right]^{1 / 2}=\frac{3}{2} \frac{\tilde{h}}{\tilde{v}_{0}}\left(\tilde{e}^{2}-\tilde{h}^{2}\right)^{1 / 2} \eta_{ \pm} .
$$

In all these expressions we have neglected terms higher order in $\tilde{i}$.

In the two-body approximation that we adopt here gravitational interaction of planetesimals changes the straight line trajectory into a hyperbola defined as

$$
r=\frac{l \cos \theta}{\sin \theta+\cos f}, \quad \tan \theta=\frac{1}{l v_{0}^{2}},
$$

where $2 \theta$ is the bending angle of the trajectory (angle between the incoming and outgoing asymptotes of the orbit) and $f$ is the true anomaly of the orbit (angle between the line of focii and a particular point on a hyperbola), varying from $\pi / 2+\theta$ (incoming) to $-\pi / 2-\theta$ (outgoing). It is trivial to show that in $(x, y, z) \equiv\left(x_{1}, x_{2}, x_{3}\right)$ coordinates this hyperbola can be represented as

$$
x_{i}=r\left[\frac{x_{c, i}}{l} \cos (f-\theta)-\frac{v_{0, i}}{v_{0}} \sin (f-\theta)\right], \quad i=1,2,3 .
$$

Also, conservation of angular momentum allows one to relate $f$ and $t$ via

$$
\frac{d f}{d t}=\frac{l v_{0}}{r^{2}}
$$

To compute the changes of orbital elements from equations (10)-(15) we also adopt approximation of "instantaneous interaction" meaning that we keep time $t$ fixed (and equal to $t_{c, \pm}$ ) throughout the scattering process. This approximation works well in high velocity encounters like the ones we are considering here because the interaction time is short. It allows us to integrate equation (14) as follows (and all others in analogous fashion):

$$
\Delta \tilde{i}_{1} \approx-\cos t_{c} \int_{-\infty}^{\infty} \frac{\partial \phi}{\partial \tilde{z}} d t=-\frac{\cos t_{c}}{l \tilde{v}_{0}} \int_{-\pi / 2-\theta}^{\pi / 2+\theta} r^{2} \frac{\partial \phi}{\partial \tilde{z}} d f
$$

where we choose a value of $\tau_{c}^{ \pm}$closest $^{8}$ to a given value of $\tau$ and then select a value of $t_{c}$ corresponding to $\tau_{c}$, see equation (A1). Then, using equations (A1)- (A8) one finds that

$$
\begin{aligned}
& \Delta \tilde{e}_{x} \approx-\sum_{n= \pm}\left(\sin t_{c}^{n} g_{1}^{n}+2 \cos t_{c}^{n} g_{2}^{n}\right), \quad \Delta \tilde{e}_{y} \approx \sum_{n= \pm}\left(\cos t_{c}^{n} g_{1}^{n}-2 \sin t_{c}^{n} g_{2}^{n}\right) \\
& \Delta \tilde{i}_{x} \approx-\sum_{n= \pm} \cos t_{c}^{n} g_{3}^{n}, \quad \Delta \tilde{i}_{y} \approx-\sum_{n= \pm} \sin t_{c}^{n} g_{3}^{n} \\
& \Delta \tilde{h} \approx-2 \sum_{n= \pm} g_{2}^{n}, \quad g_{i}^{n}=2 \frac{\tilde{v}_{i}^{n}+\tilde{x}_{i}^{n} \tilde{v}_{0}^{3}}{1+\left(\tilde{l}^{n} \tilde{v}_{0}^{2}\right)^{2}}
\end{aligned}
$$

8 Ambiguity in the choice of the origin of our $\tau$-expansion arises when $\left|\tau-\tau_{c}^{+}\right| \sim\left|\tau-\tau_{c}^{-}\right|$. However, trajectories corresponding to these values of $\tau$ do not produce noticeable contribution to the scattering coefficients. 
where $i=1,2,3$ stands for $x, y, z$, correspondingly.

In Figure 8 we compare analytical and numerical results for the changes of various orbital elements in the thin-disk limit $\tilde{i} \ll 1 \ll \tilde{e}$, which is of interest for us here. As has been previously shown by Tanaka \& Ida (1996), analytical results match numerical ones for most values of $\tau$ if one shifts the origin of $\tau$ by a small amount $d \tau \ll 1$. This shift arises from the distant interaction of the two planetesimals before they have experienced close encounter. Such a shift in $\tau$ does not affect in any way our calculation of scattering coefficients averaged over $\tau$.

A striking feature of Figure 8 is the existence of narrow intervals of $\tau$ in which numerical results strongly deviate in seemingly chaotic fashion from the analytical ones. Deviations of $\Delta \tilde{e}$, can be of order $\Delta \tilde{e}$ itself, but the discrepancy between the numerical and analytical $\Delta \tilde{i}$ exceeds analytical value of $\Delta \tilde{i}$ by orders of magnitude for some values of $\tau$. The implications of these deviations are discussed in more detail in $\$ 4$.

Neglecting for now this additional complication we average expressions (A9)-A10) over $\omega$ and $\tau$ and finally arrive at equations (25)-(28).

\section{ROLE OF DISTANT ENCOUNTERS.}

Let $\omega_{0}$ be the initial relative vertical phase of two planetesimals at infinity and $\omega$ be the value of this phase right before the close encounter. Their difference $\delta \omega_{\text {dist }}=\omega-\omega_{0}$ is small but nonzero because of the distant interaction of planetesimals preceding their close encounter. Previously we assumed $\omega$ to be equal to $\omega_{0}$ (i.e. $\left.\delta \omega_{\text {dist }}=0\right)$ thus neglecting the effect of distant interaction. Let us now see how the fact that $\delta \omega_{\text {dist }} \neq 0$ affects calculation of $\langle\tilde{\mathbf{i}} \cdot \Delta \tilde{\mathbf{i}}\rangle_{\omega}$. Using equations (A2), (A33), A10 , (A11) we find

$$
\tilde{\mathbf{i}} \cdot \Delta \tilde{\mathbf{i}}=\tilde{i} \cos \omega_{0} \Delta \tilde{i}_{x}+\tilde{i} \sin \omega_{0} \Delta \tilde{i}_{y}=-2 \tilde{i}^{2} \sum_{n= \pm} \frac{\cos \left(t_{c}^{n}-\omega\right) \cos \left(t_{c}^{n}-\omega_{0}\right)+\tilde{v}_{0}^{3} \sin \left(t_{c}^{n}-\omega\right) \cos \left(t_{c}^{n}-\omega_{0}\right)}{1+\left(l^{n} \tilde{v}_{0}^{2}\right)^{2}} .
$$

If we now average this expression over $\omega_{0}$ we find (recall that $l^{ \pm}$and $\tilde{v}_{0}$ are virtually independent of $\omega_{0}$ in the thin-disk regime when $\tilde{i} \ll \tilde{e})$

$$
\langle\tilde{\mathbf{i}} \cdot \Delta \tilde{\mathbf{i}}\rangle_{\omega} \approx-\tilde{i}^{2} \sum_{n= \pm} \frac{1-2 \tilde{v}_{0}^{3} \delta \hat{\omega_{\text {dist }}}}{1+\left(l^{n} \tilde{v}_{0}^{2}\right)^{2}}
$$

where

$$
\delta \hat{\omega_{\text {dist }}}=\frac{1}{2 \pi} \int_{0}^{2 \pi} \delta \omega_{\text {dist }}\left(\omega_{0}\right) \cos ^{2}\left(t_{c}^{n}-\omega_{0}\right) d \omega_{0}
$$

If distant interaction prior to encounter were not taken into account then $\delta \omega_{\text {dist }}=0$ and equation (B2) would be missing the second term in the numerator because averaging over $\omega_{0}$ would kill this term completely. However, when distant interaction and the possibility of non-zero $\delta \omega_{\text {dist }}$ are allowed for the omission of the second term may not be justified even if $\left|\delta \omega_{\text {dist }}\right| \ll 1$ because $\tilde{v}_{0}^{3} \gg 1$ in the situation that we consider. Then it may be possible that the product $\tilde{v}_{0}^{3} \delta \hat{\omega_{\text {dist }}} \gtrsim 1$ and dominates the numerator of equation (B2), which makes our neglect of distant interaction in calculation of $\langle\tilde{\mathbf{i}} \cdot \Delta \tilde{\mathbf{i}}\rangle_{\omega}$ unjustified. Thus, distant interaction of planetesimals can indeed explain the discrepancy between analytical and numerical values of $\langle\tilde{\mathbf{i}} \cdot \Delta \tilde{\mathbf{i}}\rangle_{\omega}$ observed in Figure 12 . 